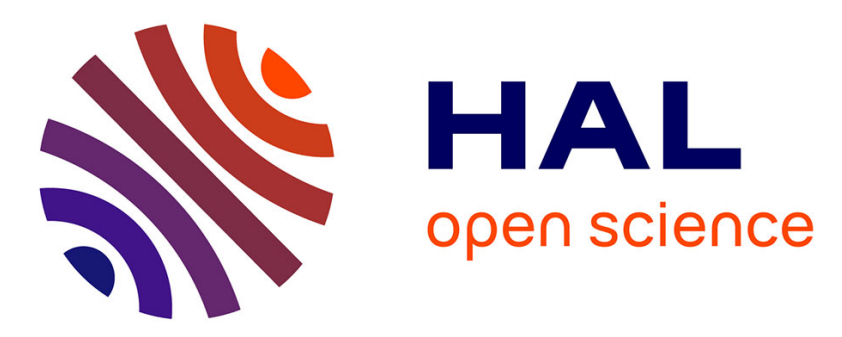

\title{
Mimicking cortex convolutions through the wrinkling of growing soft bilayers
}

Martine Ben Amar, Adrien Bordner

\section{To cite this version:}

Martine Ben Amar, Adrien Bordner. Mimicking cortex convolutions through the wrinkling of growing soft bilayers. Journal of Elasticity, 2017, pp.1-26. 10.1007/s10659-017-9622-9 . hal-01452908

\section{HAL Id: hal-01452908 \\ https://hal.sorbonne-universite.fr/hal-01452908}

Submitted on 2 Feb 2017

HAL is a multi-disciplinary open access archive for the deposit and dissemination of scientific research documents, whether they are published or not. The documents may come from teaching and research institutions in France or abroad, or from public or private research centers.
L'archive ouverte pluridisciplinaire HAL, est destinée au dépôt et à la diffusion de documents scientifiques de niveau recherche, publiés ou non, émanant des établissements d'enseignement et de recherche français ou étrangers, des laboratoires publics ou privés. 


\title{
Mimicking cortex convolutions through the wrinkling of growing soft bilayers
}

\author{
Martine Ben Amar and Adrien Bordner
}

Received: date / Accepted: date

\begin{abstract}
Brain convolutions are a specificity of mammals. Varying in intensity according to the animal species, it is measured by an index called the gyrification index, ratio between the effective surface of the cortex compared to its apparent surface. Its value is close to 1 for rodents (smooth brain), 2.6 for newborns and 5 for dolphins. For humans, any significant deviation is a signature of a pathology occurring in fetal life, which can be detected now by magnetic resonance imaging (MRI). We propose a simple model of growth for a bilayer made of the grey and white matter, the grey matter being in cortical position. We analytically solved the neo-Hookean approximation in the short and large wavelength limits. When the upper layer is softer than the bottom layer, the selection mechanism is shown to be dominated by the physical properties of the upper layer. When the anisotropy favors the growth tangentially as for the human brain, it decreases the threshold value for gyri formation. The gyrification index is predicted by a post-buckling analysis and compared with experimental data. We also discuss some pathologies in the model framework.
\end{abstract}

Keywords Brain · Gyrification index · Buckling · Post-buckling · Finite Elasticity · Neo-Hookean $\cdot$ Surface tension

\section{Introduction}

This paper intends to clarify the onset of buckling of a bilayer during growth. Indeed, if the one layer linear mode of instability is well understood since the pioneering work of Biot [11] concerning soft hyperelastic materials, the theoretical prediction of the wavelength remains matter of debates, especially for bilayers. It is also true that the wavelength selection for one layer is not obvious: the linear analysis predicts a threshold but at vanishing wavelength or infinite wavenumber, a result which contradicts experimental observations. Then, the wavelength selection at threshold is a matter of a not trivial singular perturbation. In the case of a

Martine Ben Amar and Adrien Bordner

Laboratoire de Physique Statistique, Ecole Normale Supérieure, PSL Research University; Sorbonne Universités UPMC Univ Paris 06; CNRS; 24 rue Lhomond, 75005 Paris, France first address

Institut Universitaire de Cancérologie, Faculté de médecine, Université Pierre et Marie Curie-Paris 6, 91 Bd de l'Hôpital, 75013 Paris, France second address 
bilayer, the multiplicity of independent parameters of the top layer 1 versus the bottom layer 2 may give some hope for a simpler determination of the wavelength. Differential growth between both layers, the ratio $\mu$ of shear moduli (defined by $\mu=\mu_{1} / \mu_{2}$ ) or the two different thicknesses represent new degrees of freedom that can modify the wavelength selection, compared to the one layer model. In fact, from previous works by Biot [10], achieved several years before [11], the wrinkling of a bilayer, made of a thin film on top of an infinite soft substrate, under external compression, can be simply explained by the Hookean elasticity of the stiff thin plate modified by a local contribution due to the substrate which reminds of the Winkler foundation model. According to Biot's result [10], it gives an instability with a critical pressure threshold which varies in $\mu^{-2 / 3}$, a selected wavelength $\lambda$ at threshold in $\mu^{1 / 3}$ which is large since the dimensionless ratio $\mu$ is a large dimensionless number in this limit. This situation of an infinite soft substrate falls in the domain of long wavelengths while the Biot's singularity of a neo-Hookean monolayer concerns the short-wavelength limit.

Morphogenesis and embryogenesis reveal a lot of examples of bilayer wrinkling due to growth such as our fingerprints [37,4,19], intestine villi [29,8], esophagus [39] and brain convolutions $[56,5,28]$. Occurring in fetal life, at a rather precise stage post fertilization, these instabilities are common to most mammals, but not only, and concern differential growth of bilayers, with a bottom layer, in most cases, stiffer than the upper layer.

The aim of this paper is then to clarify this problem of wavelength selection with a special focus on brain convolutions. Our analysis consists of a minimal set of mechanical parameters to describe the growth of a planar bilayer: both layers of different thicknesses are represented by the neo-Hookean elasticity with different shear moduli, the growth in each layer is treated with the multiplicative gradient decomposition formulation [48]. The upper layer is free, the bottom layer infinite or attached to a stiff substrate. Our purely analytical treatment is limited to harmonic patterns derived close to the stability limit, in contrast to more elaborate numerical investigations where detailed phase-diagrams are derived in the nonlinear regime of parameters [60]. The chosen formulation [48] treats growth in the same way as initially pre-strained materials which, next, are remodeled under physical constraints, such as the minimization of the elastic energy and the boundary conditions. Most of these simulations are based on this analogy. Comparison can be achieved with our results if the growth and the pre-strain per unit volume correspond. The advantage of numerical investigations $[13,34,60]$ is the possibility to detect non harmonic patterns, which is impossible here.

The two limiting cases, short and long wavelengths, are examined for completeness, although the brain is mostly concerned by the short wavelength limit. The gyrification index is analytically predicted. The paper is organized as follows: Section 2 is devoted to a short overview of the brain in fetal life. Section 3 presents the biomechanical model and the exact results for the threshold determination for a growing bilayer. Section 4 focusses on the short wavelength determination and by a nonlinear analysis, Section 5 shows that for the physical parameters concerning the brain, the bifurcation is supercritical allowing the determination of the gyrification index that we compare to experiments $[68,1]$. In Section 6 , the long wavelimit is recovered and is compared to the Biot's result [10] in the case of growth. Finally in Section 7, we conclude that to some extent, the bilayer model of soft nonlinear elasticity with growth explains the onset of gyri formation of the brain. 


\section{The brain convolutions, the gyrification index and associated pathologies}

We can distinguish mammals in two groups according to the wrinkling level of the brain. Smooth brains are called lissencephalic (and concern mostly rodents) whereas folded brain are gyrencephalic. The human brain is formed of two hemispheres, highly convoluted, but less than the lama, the dolphin, etc. At macroscopic scales, the hemispheres are composed of two main layers: the grey matter and white matter. The grey matter forms the cortex and is located below the skull and the meninges. It contains the neuronal bodies and the glial cells (which are cells of support). The axons and dendrites (very long extension of the neural cells) are radially oriented, then inducing anisotropy in the brain tissue. The white matter is mainly composed of glial cells and a high number of myelinated axons which transmit long distance signals between the cortex and the other brain regions.

In the fetal life, the brain originates from a smooth and homogeneous structure named telencephalon which appears at months 2-3 of gestation, for humans. During months 3-4, the neuron precursors proliferate in a thin zone at the basis of telencephalon (the subventricular zone). Then it follows a radial migration of these cells resulting in the formation of the cortical layer (grey matter). The timing and the direction of this neuronal migration is crucial, and any perturbation of these processes leads to major disorders and folding abnormalities in the newborn brain [59]. The migration is under the high control of radial glial cells, forming a radial scaffold along which the neurons migrate. Although oriented radially, these cells also deviate at the level of the cortex, and contribute to the tangential growth of the cortex as shown recently [47]. The meninges are thought to play here a crucial role by secreting and organizing the pial basement membrane which makes tight contact with the cortical layer. The radial glial cells are anchored to this membrane and any defect of the meninges disrupts the scaffold leading to malformations [50]. Once neuronal migration ends at month 4, the growth of the brain really begins, together with the surface buckling and the first cerebral indentations (sulci): this is called gyrification. The gyrification coincides with the period of highest tissue growth [61].

Recently, mechanical measurements have been performed on brain tissues and have shown that these two layers have different mechanical properties induced by the various micro-structures. Experimental results showed that the white matter is stiffer than the gray matter for adults and fetuses [64,33,5]. In Table 1, recent data measurements are reported for mammals, showing a noticeable dispersion on experimental values which are strongly dependent on the technique, the data analysis, the regional variation but also on the age of the brain and the strain intensity. Some consensus appears for a stiffness ratio $\mu$ of order $0.7[55,64]$ except perhaps for rodents. Rodent cerebellum seems to be in opposition with $\mu=1.5$, larger than one [22]. However, if one must be cautious about cerebellum and cerebrum data comparisons, it is important to recall that most of rodents do not have gyri. For human brain, this consensus is based on experimental biomechanical measurements [64,33, $5]$ and on analytical works on wrinkling $[64,55]$. However, during the final revision of this work, an extensive experimental study concerning mechanical tests in static and dynamics [14] announces opposite conclusions concerning the stiffness of the cortex and of the white matter. Achieved on human cadavers, with an average age of 66 years after autopsy, these authors study the human brain elasticity and viscoelasticity in vitro in various location such as the corpus callosum, the corona radiata but not immediately below the cortex, that is in the sub-plate. For various loading conditions: tension, compression and shear, they conclude that the cortex always appears stiffer than the white matter, in opposition with other results [33]. Clearly the question of the comparison of the stiffness of both layers: the cortex and the white matter, so important for the modeling, is matter of debate. In any case, we must 
Table 1 BRAIN STIFFNESS

\begin{tabular}{llllll}
\hline \hline & $\begin{array}{l}\text { Bovine }^{1} \\
\text { cerebrum }\end{array}$ & $\begin{array}{l}\text { Bovine }^{\mathbf{2}} \\
\text { cerebrum }\end{array}$ & $\begin{array}{l}\text { Pig }^{1} \\
\text { cerebrum }\end{array}$ & $\begin{array}{l}\text { Pig }^{1} \\
\text { cerebrum }\end{array}$ & $\begin{array}{l}\text { Rat }^{3} \\
\text { cerebellum }\end{array}$ \\
\hline Grey matter $(\mathrm{kPa})$ & 0.689 & 1.5 & 2.23 & 1.195 & 0.454 \\
White matter $(\mathrm{kPa})$ & 1.440 & 2 & 3.083 & 1.787 & 0.29 \\
ratio $\mu$ & 0.48 & 0.75 & 0.72 & 0.64 & 1.565 \\
Reference & {$[12]$} & {$[12]$} & {$[58]$} & {$[35]$} & {$[22]$} \\
\hline
\end{tabular}

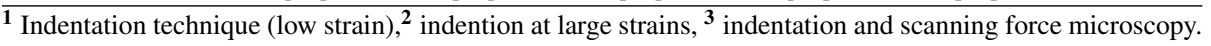

keep in mind that the gyrification process we examine here concerns embryonic stages so fetuses and not cadavers of old human beings. As underlined in [64], the stiffness of the brain, whatever the anatomic regions, increases with aging and it seems that it increases more for the cortex [64]. As also mentioned in [64], when experimental data are lacking or debatable, a theoretical treatment may help to clarify the situation.

These properties motivate our two-layer model and explain why we will focus our study on the shear moduli ratio $\mu$. Many models have been proposed to explain the brain folding $[42,52]$. It first has been suggested that the considerable growth of the telencephalon, under the external constraint of the limited volume by the cranium, is responsible for gyrification ("crumpled sheet model")[18]. However, clinical and animal experiments have shown that the cranium constraint is a minor factor for buckling [3,26]. Another model [42] treats the cortex as a one smectic layer subject to axons pulling from the white matter. According to the last biomechanics review [28], two hypotheses emerge and are in competition, differential growth between both layers and mechanical forces such as axonal tension. Although it remains controversial, this last hypothesis disagrees with dissection experiments [64]. Knowing that the cortical surface area increases faster than the brain volume has inspired numerical models [56,54]. It is shown that the tangential expansion of an outer layer, bounded to a more slowly growing central core, induces buckling. In addition, cortical development studies reveal unequal cell migration mechanisms for radial and tangential expansion inducing anisotropy in the cortical layer growth [47]. Then, we can conclude that the human cortex growth of healthy beings is anisotropic and this anisotropy will be introduced in the growth representation in our two-layer model.

Many pathologies are now related to cerebral folding. Recent observations link autistic disorders to abnormal deepness of the cerebral sulci [15], affecting the social communication functions. Moreover, the abnormal regulation of the brain growth results in an early overgrowth followed by an abnormally slow-down during development [21]. This increase concerns the cortical area rather than the cortical thickness [44]. It also exists rare generalized abnormalities of gyrification, for instance the lissencephaly which consists in a low number of sulci and, on contrary, polymicrogyria where the gyri are too many and too small. Physiopathology of these diseases is mainly unknown, but neuronal migration and the failure of cell proliferation inducing abnormal growth could be involved [23,43]. It is also hypothesized that the thickened meninges potentially influence folding and could lead to polymicrogyria [51].

Our understanding of the growth dynamics of the brain in fetal life will increase in the next future as the same time as its connection with pathologies $[25,38]$. However, the simple buckling of a double elastic layer of soft tissues is itself rather poorly understood and many works accept wavelength relationships out of their domain of application. Some analytical results such as the wavelength varying like a power law of the ratio of both stiffnesses [10] are very simple, but not always used in the range of physical parameters where they are valid. 
In addition, even if there is a difference between mechanical properties or growth dynamics for the white matter and grey matter, we cannot expect large differences in physical properties, which mostly prohibit the use of limiting models in nonlinear elasticity. Focussing only on the brain morphogenesis, the main ingredients seem to be the ratio between the shear modulus of the two layers $\mu$ (grey matter versus white matter shear modulus) but remaining of order one, a growth anisotropy which favors tangential growth in the cortex and a differential growth between both layers. In addition the cortex is much thinner that the white matter so we will pay more attention of the case of infinite substrate. We introduce these ideas in the following section.

\section{The model}

We consider two growing layers of soft materials, joined together, having different thicknesses. Such ideal situation is known to generate buckling, folding, creases $[57,31,60]$ and even other more complex patterns $[2,60,8]$, especiallly in three dimensions, as soon as the growth differs in the two layers. Complex patterns are out of reach of the linear analysis presented here and they require sophisticated numerical treatments. However, most of them are issued of the linear regime presented here and fall in the nonlinear regime of the first bifurcation. The first bifurcation,which is analyzed here, only allows to explain the existence of the wrinkling in function of the physical parameters and the pattern length scale. Since both layers are strongly connected, axial growth in the vertical direction induces a configuration of lowest elastic energy. This geometry differs from simulations based on prestretched substrate [60] in the horizontal direction which may lead to different conclusions, although, at first sight, both problems are similar. To limit the number of independent parameters, the growth is assumed homogeneous in both samples, anisotropic in the upper layer, the materials are considered as hyperelastic and neo-Hookean $[45,30]$ and the horizontal dimensions infinite in both directions. The upper layer, called 1 in the following, has a thickness $H$, while, the thickness of the substrate 2 is either infinite or chosen as unity. The shear modulus of the substrate is also chosen as unity and we call $\mu$ the ratio between the shear modulus of the upper slab compared to the substrate one. These hypotheses can appear rather restrictive to fully represent the biological complexity of growth during morphogenesis or embryogenesis, however a growing number of independent parameters obscures the wrinkling results, changing the threshold value for existence without always changing the physical reality. Here we mostly focus on the ratio between both shear moduli which is often smaller than one in biological systems $[19,8,56]$ contrary to material sciences $[66,36,65]$. Capital letters $(X, Y)$ represent the cartesian coordinates of a point in the reference configuration, assumed free of stresses at initial time, and, small letters $(x, y)$ are devoted to the current configuration, at time t. Both samples are assumed incompressible.

\subsection{The growth model}

Assuming the classical multiplicative decomposition [48], growth is represented by a tensor $\mathbf{G}$, the geometric deformation gradient tensor by $\mathbf{F}$ such as $\mathbf{F}=\mathbf{F}_{\mathbf{e}} \cdot \mathbf{G}$, where $\mathbf{F}_{\mathbf{e}}[30,45]$ is the elastic deformation gradient tensor. Once the hypothesis that the characteristic mechanical time is much shorter compared to the typical time scale of growth processes, one can neglect the dynamics and determine the current configuration at time $t$ by a variational principle of minimization of the elastic energies. 

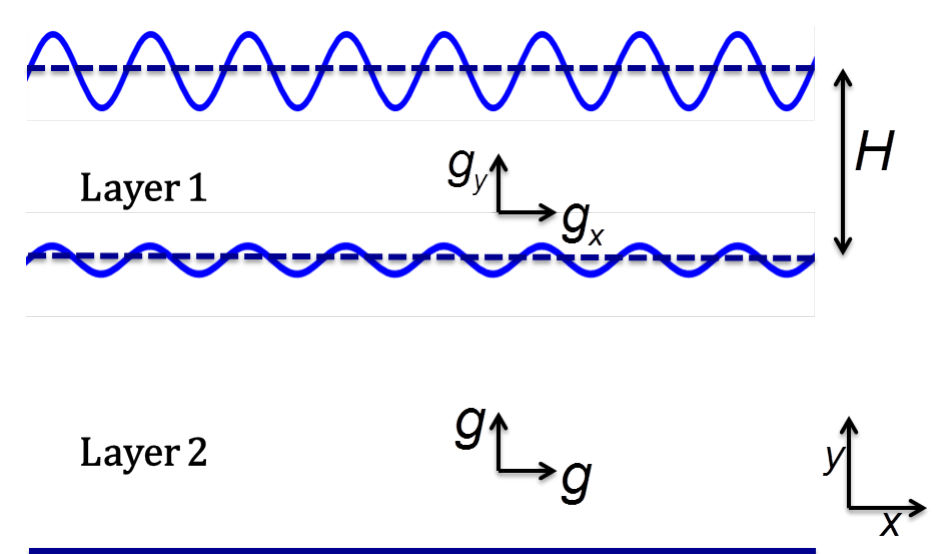

Fig. 1 The bilayer model under buckling.

The growth tensor will be chosen anisotropic in the upper layer with an eigenvalue $g_{x}$ along the $x$ direction, tangent to the interface, larger than the one $g_{y}$ along the perpendicular direction. This will reflect the structure of the grey matter and its tendency to proliferate more in the direction parallel to the cranium. This growth anisotropy is not known experimentally but may have some consequences on final patterns at large strains, as shown for villi [8]. For the white matter, there is no reason to introduce such difference and we will choose an isotropic growth modeling, to limit the number of parameters. The growth of our bilayer is then characterized by three eigenvalues. The growth tensors $\mathbf{G}_{i}$ and elastic tensors $\mathbf{F}_{e_{i}}$ are then:

$$
\mathbf{G}_{1}=\left[\begin{array}{cc}
g_{x} & 0 \\
0 & g_{y}
\end{array}\right], \mathbf{G}_{2}=\left[\begin{array}{ll}
g & 0 \\
0 & g
\end{array}\right], \mathbf{F}_{e_{1}}=\left[\begin{array}{ll}
g_{x}^{-1} x_{, X} & g_{y}^{-1} x_{, Y} \\
g_{x}^{-1} y_{, X} & g_{y}^{-1} y_{, Y}
\end{array}\right], \mathbf{F}_{e_{2}}=\left[\begin{array}{ll}
g^{-1} x_{, X} & g^{-1} x_{, Y} \\
g^{-1} y_{, X} & g^{-1} y_{, Y}
\end{array}\right]
$$

Where the notation with a comma in subscript means partial derivative. Hereafter we use the index 1 and 2 when it is necessary to distinguish the layers. We drop the index otherwise. An index with a coma indicates a partial derivative. In the following, we will assume that fiber-like structures such as axons or vessels, present in the brain, do not contribute to an anisotropic mechanical behavior. Indeed, Budday et al. [14] have extensively analyzed this question experimentally in various parts of the white matter, especially the corpus callosum, the most fibrous area of the human brain and they conclude that, from the biomechanical viewpoint, the white matter can be represented as the cortex by an isotropic constitutive law. In addition we assume that the brain is incompressible which means that any change of volume is due to cell proliferation and not to elastic stresses. Then we will adopt the constraint of incompressibility and the relative volume variation $J$ is given by: $J=\operatorname{det} \mathbf{F}=$ $\operatorname{det} \mathbf{G}$ so $J_{1}=g_{x} g_{y}$ and $J_{2}=g^{2}$. These relations are automatically satisfied by the definition of stream-functions [6], as shown in the next section. Before going further, let us compare our model with the pre-strain method used in numerical simulations [60]. Considering a sample of initial length $L_{s}$ for the substrate and $L_{f}$ for the upper layer, the process consists in extending first the substrate from $L_{s}$ up to $L_{f}$, then in gluing both samples up to a final state which, in absence of buckling, leads to a final length $L$ for the bilayer. It gives the following elastic deformation gradients which can be compared to our uniaxial growth model along 
the $Y$ direction:

$$
\mathbf{F}_{e f}=\left[\begin{array}{cc}
L / L_{f} & 0 \\
0 & L_{f} / L
\end{array}\right] ; \mathbf{F}_{e 1}=\left[\begin{array}{cc}
1 / g_{x} & 0 \\
0 & g_{x}
\end{array}\right] ; \quad \mathbf{F}_{e s}=\left[\begin{array}{cc}
L / L_{s} & 0 \\
0 & L_{s} / L
\end{array}\right] ; \mathbf{F}_{e 2}=\left[\begin{array}{cc}
1 / g & 0 \\
0 & g
\end{array}\right]
$$

In this simplified configuration, the unknown $L$ value is derived from the bilayer elastic energy minimization and in the limit of thin substrate gives:

$$
L \sim L_{s}+\mu H \frac{L_{f}^{4}-L_{s}^{4}}{4 L_{f}^{2} L_{s}}
$$

while the compressive strain, used as a control parameter is $\varepsilon=\left(L_{f}-L\right) / L_{f}=1-1 / g_{x}$. At this stage, it is then possible to compare both approaches.

\subsection{The definition of non linear stream functions}

One way to impose the constraint of incompressibility consists in introducing a Lagrange parameter called the pressure. Another way consists in minimizing the elastic energy in the sub-set of deformations which automatically satisfy the incompressibility constraint and preserve the area in two dimensions. The construction of such deformations proposed by Carroll [17], in elasticity, is based on the mixed coordinate systems where one coordinate is chosen in the reference frame and the other in the current configuration [6]. The advantage of such representation is to eliminate the pressure which, otherwise, remains an unknown function coupled to the deformations and to perform the variation with respect to a unique function called the stream function by analogy with the Eulerian hydrodynamic formulation for Darcy and Stokes flows in two dimensions. Notice that this method can be applied to any imposed preserving area mapping between the initial and current configuration, once it remains only dependent on a unique coordinate so it can be extended to the radial geometry [63]. We then introduce two stream functions $\Phi$ and $\Psi$ [6] which are defined by the following implicit relationships involving a mixed coordinate representation:

$$
X_{1}=\frac{1}{J_{1}} \frac{\partial \Phi\left(x_{1}, Y_{1}\right)}{\partial Y_{1}} ; y_{1}=\frac{\partial \Phi\left(x_{1}, Y_{1}\right)}{\partial x_{1}} ; X_{2}=\frac{1}{J_{2}} \frac{\partial \Psi\left(x_{2}, Y_{2}\right)}{\partial Y_{2}} ; y_{2}=\frac{\partial \Psi\left(x_{2}, Y_{2}\right)}{\partial x_{2}}
$$

In the following, notations for partial derivatives with respect to coordinates $\left(x_{i}, Y_{j}\right)$ will be also defined by a subscript with a coma followed by $x_{i}$ and/or $Y_{j}$. The components of the geometric deformation gradient tensor read:

$$
\left\{\begin{array}{l}
\left.\frac{\partial x}{\partial X}\right|_{Y}=J \Phi_{, x Y}^{-1},\left.\quad \frac{\partial y}{\partial Y}\right|_{X}=\left(\Phi_{, x Y}^{2}-\Phi_{, x x} \Phi_{, Y Y}\right) \Phi_{, x Y}^{-1} \\
\left.\frac{\partial x}{\partial Y}\right|_{X}=-\Phi_{, Y Y} \Phi_{, x Y}^{-1},\left.\quad \frac{\partial y}{\partial X}\right|_{Y}=\Phi_{, x x} \Phi_{, x Y}^{-1}
\end{array}\right.
$$

We can now proceed to the variational calculation. 


\subsection{Variational calculation}

The mechanical energy of the bilayer is the sum of the elastic energies of both layers $E_{i}$ and the interfacial energy at the frontier between them, $E_{c 2}$, and on top of the upper layer $E_{c 1}$. We choose the simplest hyperelastic elastic model, the neo-Hookean one $[45,30]$, for both layers which reads:

$$
E_{1}=\frac{1}{2} J_{1} \mu \int_{0}^{H} d X d Y \operatorname{Tr}\left(\mathbf{F}_{\mathbf{e}} \cdot \mathbf{F}_{\mathbf{e}}^{\mathbf{T}}-2 \mathbf{I}\right) \quad \text { and } \quad E_{2}=\frac{1}{2} J_{2} \int_{-H_{2}}^{0} d X d Y \operatorname{Tr}\left(\mathbf{F}_{\mathbf{e}} \cdot \mathbf{F}_{\mathbf{e}} \mathbf{T}^{\mathbf{T}}-2 \mathbf{I}\right) .
$$

Eventually, $\mathrm{H}_{2}$ can be considered as infinite, and

$$
E_{c_{i}}=d_{i} \int_{Y=I n t} d x \sqrt{\left(\frac{\partial x}{\partial X}\right)^{2}+\left(\frac{\partial y}{\partial X}\right)^{2}} .
$$

As before, $\mu=\mu_{1} / \mu_{2}$ and $d_{i}$ represents the capillary parameter of the upper layer or of the intermediate interface between both layers. Replacing the elastic tensor components by the partial derivatives of the stream function for the upper layer reads :

$$
E_{1}=\frac{1}{2} \mu g_{y}^{2} \int_{0}^{H} d x d Y \Phi_{, x Y}^{-1}\left(1+\Phi_{, x x}^{2}+g_{y}^{-4}\left[\Phi_{, Y Y}^{2}+\left(\Phi_{, x Y}^{2}-\Phi_{, x x} \Phi_{, Y Y}\right)^{2}\right]-2 g_{y}^{-2} \Phi_{, x Y}^{2}\right),
$$

and a similar relationship for $E_{2}$ involving $\Psi$. A trivial configuration can be found where the growth is forced in the $Y$ direction which also satisfies the constraints on the stresses at the boundaries. This basic state corresponds to a thickening of both layers and the stream functions can be decomposed into two parts according to:

$$
\Phi(x, Y)=x Y g_{x} g_{y}+\varepsilon \phi(x, Y) \text { and } \Psi(x, Y)=x Y g^{2}+\varepsilon \psi(x, Y) .
$$

The first term represents a purely axial growth. In the following, the elastic energies will be expanded up to fourth order in power of $\varepsilon: E_{i}=E_{0}+\varepsilon^{2} E_{i}^{(2)}+\varepsilon^{4} E_{i}^{(4)}+o\left(\varepsilon^{4}\right)$. The derivation of the Euler-Lagrange equation and the bifurcation analysis are achieved at second order of the energy expansion but the post-buckling treatment requires the expansion up to fourth order, see Section 5. Once $\phi$ and $\psi$ are expanded in Fourier modes $F(Y) \sin (k x)$, these modes are uncoupled at second order but also at fourth order. Indeed a solution of non-linear equations in Fourier modes generates secular terms and a better approximation consists in the elimination of mode coupling [9]. This simplifies the analysis and gives for the upper layer:

$$
\begin{gathered}
E_{1}^{(2)}=\frac{\mu}{4 J_{1}^{2} p} \int d Y\left(J_{1}^{2} k^{4} p^{2} F_{1}^{2}+k^{2}\left(3 J_{1}^{2}+p^{2}\right) F_{1}^{\prime 2}+2 J_{1}^{2} k^{2} F_{1} F_{1}^{\prime \prime}+F_{1}^{\prime \prime 2}\right), \\
E_{c 1}^{(2)}=\frac{1}{2} d_{1} k^{3} \pi F_{1}(H)^{2},
\end{gathered}
$$

where $F^{\prime}$ (respectively $F$ ") means the first (respectively the second) derivative with respect to $Y$ and the anisotropy parameter $p$ is equal to $p=g_{y} / g_{x}$. Differential tangential expansion leads to $p<1$.

$$
\begin{gathered}
E_{1}^{(4)}=\frac{\mu k^{2}}{16 p J_{1}^{4}} \int\left(k^{2} p^{2} F_{1}^{\prime 2}\left(J_{1}^{2} k^{2} F_{1}^{2}+3 F_{1}^{\prime 2}\right)+F_{1}^{\prime \prime 2}\left(3 J_{1}^{2} k^{2} F_{1}^{2}+F_{1}^{\prime 2}\right)\right), \\
E_{c 1}^{(4)}=-\frac{3}{32} d_{1} k^{7} \pi F_{1}(H)^{4},
\end{gathered}
$$


with similar relationships for $E_{2}^{(2)}$ and $E_{2}^{(4)}$ (with $\mu=p=1$ and $J_{1}$ changed to $J_{2}$ ) for the bottom layer. The Euler-Lagrange equation is a fourth-order linear differential equation:

$$
\frac{d^{4} F(Y)}{d Y^{4}}-k^{2}\left(J^{2}+p^{2}\right) \frac{d^{2} F(Y)}{d Y^{2}}+J^{2} p^{2} k^{4} F(Y)=0
$$

with the boundary conditions explicated in the Appendix (9.1)

3.4 The profile function at linear order

When both layers grow, each solution of Eq.(14) can be written as superposition of hiperbolic functions, according to:

$$
\begin{aligned}
& F_{1}(Y)=\frac{\varepsilon}{1+A_{1}}\left(\cosh \left(J_{1} k Y\right)+A_{1} \sinh \left(J_{1} k Y\right)+A_{2} \cosh (k p Y)+A_{3} \sinh (k p Y)\right), \\
& F_{2}(Y)=\frac{\varepsilon}{1+A_{1}}\left(B_{1} \cosh \left(J_{2} k Y\right)+B_{2} \sinh \left(J_{2} k Y\right)+B_{3} \cosh (k Y)+B_{4} \sinh (k Y)\right) .
\end{aligned}
$$

Our choice for $F_{2}(Y)$ is valid for a finite thickness of the bottom layer and the boundary conditions must be prescribed for this layer too, at bottom. For a finite bilayer, we choose a condition of no-sliding of the bottom layer on a rigid support. When the second layer is infinite, one needs to change $F_{2}$ which becomes:

$$
F_{2}(Y)=\frac{\varepsilon}{1+A_{1}}\left(C_{1} \exp \left(J_{2} k Y\right)+C_{2} \exp (k Y)\right),
$$

where $Y<0$ and the interface is chosen at $Y=0$. When the second layer does not grow but serves only as a support, Eq.(16) is modified and $F_{2}$ is changed into

$$
F_{2}(Y)=\frac{\varepsilon}{1+A_{1}}\left(D_{1} \cosh (k Y)\left(1+D_{2} Y\right)+D_{3} \sinh (k Y)\left(1+D_{4} Y\right)\right) .
$$

If the second layer does not grow and is also infinite, $F_{2}$ reads:

$$
F_{2}(Y)=\frac{\varepsilon}{1+A_{1}} E_{1} \exp (k Y)\left(1+E_{2} Y\right)
$$

In Eq.(15) up to Eq.(19), $\varepsilon$ is a parameter of expansion for weakly nonlinear analysis which is evaluated in the post-buckling analysis (see Section 5). Since the amplitude of the perturbation is $\varepsilon$, one arbitrary coefficient has been fixed to 1 in $F_{1}$. However, it remains 7 coefficients to fix for 8 equations so $k$ cannot be chosen arbitrarily. The $k$ determination is a challenging problem of selection. However the ability of such models to describe the buckling is given only once $k$ is theoretically determined. Despite the simplicity of the model, explicit analytical solution cannot be found (see Appendix 9.2), but two opposite cases can be examined: a) the short wavelength limit equivalent to infinite $k(\lambda=2 \pi / k)$ and $b)$ the long wavelength limit and vanishing wavenumber $k$. Both cases have been partially studied in the past but here, we aim to clarify all possible scenarios. 


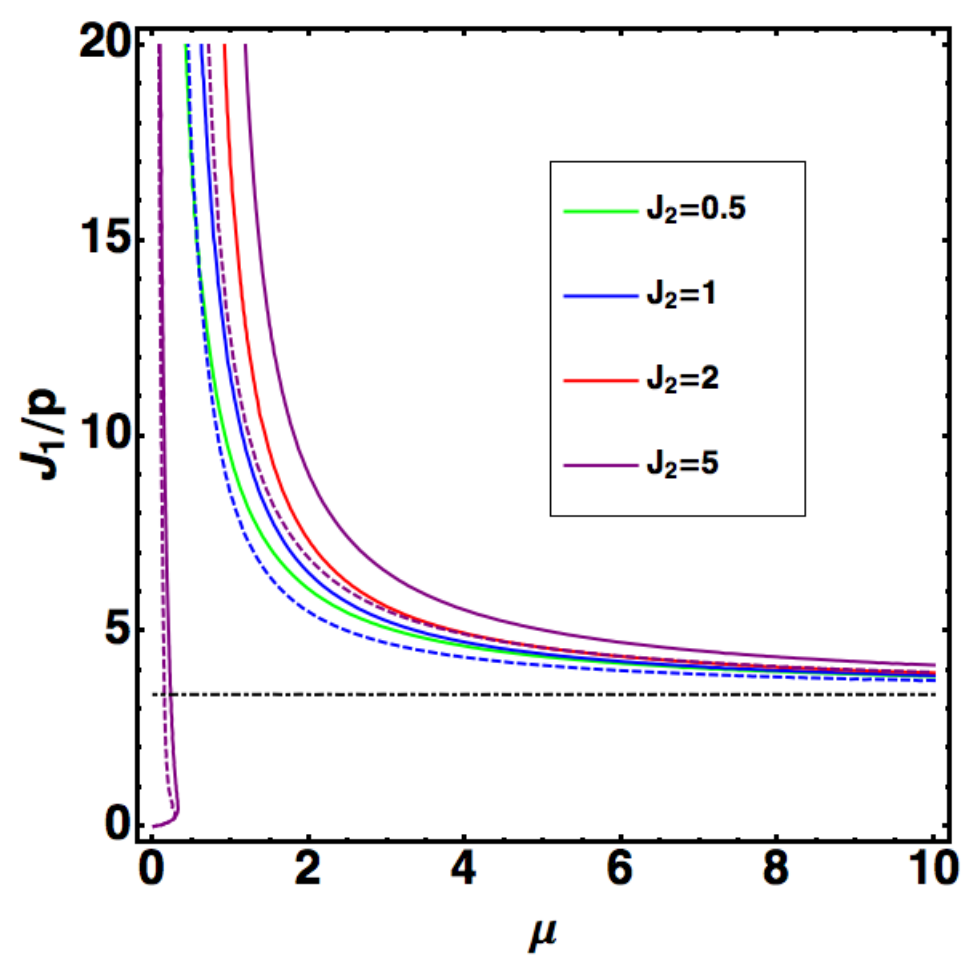

Fig. 2 Secondary bifurcation parameter $J_{1} / p$ as a function of $\mu$, ratio between both shear moduli of the upper layer versus the substrate. Continuous colored lines correspond to $p=1$, colored dashed lines $p=0.5$. As expected the growth anisotropy lowers the required growth rate. The dot-dashed line in black correspond to $J_{c} \sim 3.38$.

\section{The Dispersion relation at short-wavelengths}

The short-wavelength range concerns spatial oscillatory modes with a wavelength smaller than the lateral dimensions of the sample and all lengths such as the two thicknesses of the layer. It means that $k H$ is a priori a large dimensionless parameter. The dispersion relation results from the calculation of the coefficients defined in Eqs. $(15,16,17,18,19)$ and introduced in the boundary conditions (see Appendix 9.1). After an easy but tedious calculation, all coefficients are determined and the solvability condition, or equivalently the dispersion relation, can be written as the sum of four exponentials with rather complicated polynomial coefficients of the physical quantities introduced in the model. It is possible to solve numerically this dispersion relation, however we can go further in the analysis by noticing that, if the upper layer grows, $\left(J_{1}>1\right)$, two exponentials have the largest positive exponents and so will dominate the dispersion relation in the limit of infinite wavenumber $k$. As shown in [6], these exponentials control the selection mechanism. Once restricted to dominant quantities, the approximated dispersion relation becomes:

$$
\mathscr{D}=\mathscr{D}_{a}-\mathscr{D}_{b} e^{-2 H k p}
$$




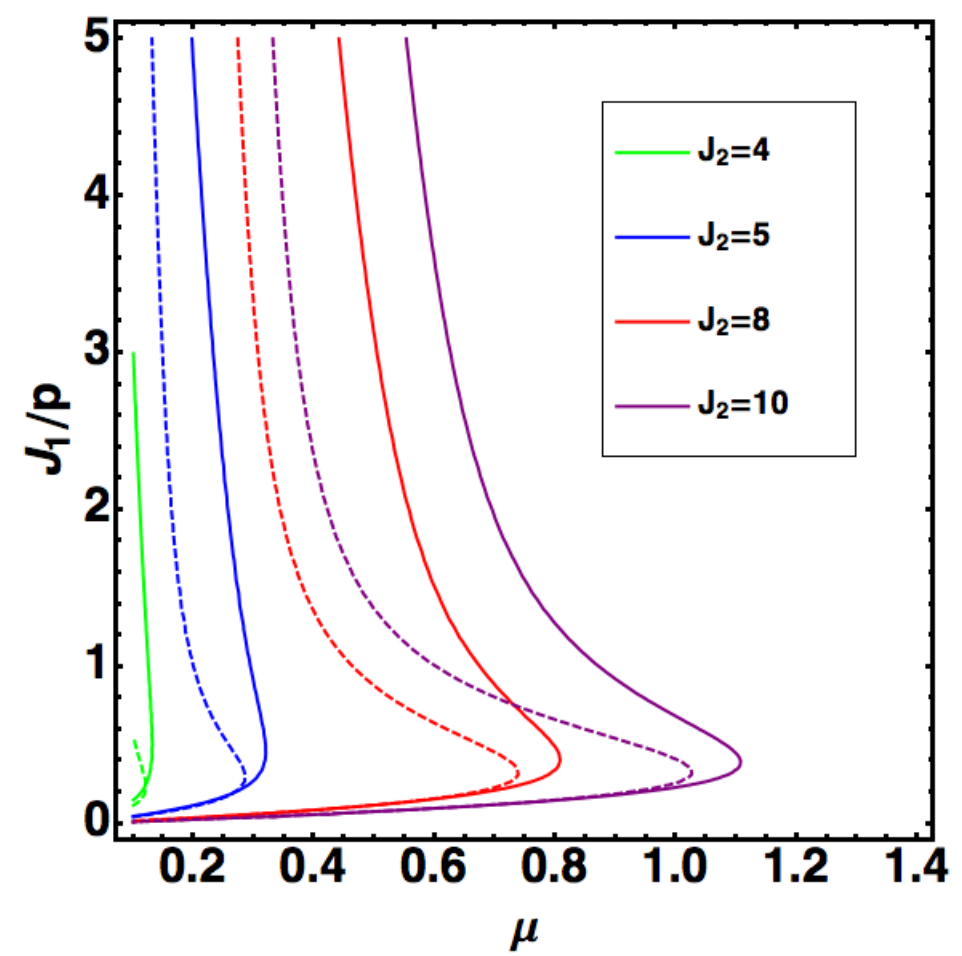

Fig. 3 Secondary bifurcation parameter $J_{1} / p$ as a function of $\mu$, ratio between both shear moduli of the upper layer versus the substrate. The substrate is stiffer than the upper layer when $\mu<1$. The growth rate in the substrate is above the critical parameter $J_{c} \sim 3.38$. Continuous colored lines correspond to $p=1$, colored dashed line $\mathrm{p}=0.5$. As expected the growth anisotropy lowers the required growth rate.

As shown in [6], for one growing layer, the key of the analysis is the existence of two polynomials that we introduce at first:

$$
\left\{\begin{array}{l}
\mathbf{P}_{0}(J)=-1-J-3 J^{2}+J^{3} \\
\mathbf{Q}_{0}(J)=1-J+3 J^{2}+J^{3}
\end{array}\right.
$$

which allows to write simply $\mathscr{D}_{a}$ and $\mathscr{D}_{b}$ for the one layer case. For a bilayer, these terms are more complicated and read:

$$
\mathscr{D}_{a}=\left(\mathbf{P}_{0}\left(\tilde{J}_{1}\right)-d_{1} k \tilde{J}_{1}\left(\tilde{J}_{1}+1\right)\right) \mathscr{P}\left(\mu, \tilde{J}_{1}, J_{2}, d_{2}\right),
$$

where $\tilde{J}_{1}=J_{1} / p$ and $\mathscr{P}$ is a polynomial of degree two in $\mu$. In the same way, we derive $\mathscr{D}_{b}$ :

$$
\mathscr{D}_{b}=\left(\frac{1+\tilde{J_{1}}}{1-\tilde{J_{1}}}\right)^{2} \mathbf{Q}_{0}\left(\tilde{J_{1}}\right) \mathscr{Q}\left(\mu, \tilde{J_{1}}, J_{2}\right),
$$

The new polynomials coming from the bottom layer are then, for $\mathscr{D}_{a}$ :

$$
\mathscr{P}=\mu^{2} J_{2}^{2}\left(\mathbf{P}_{0}\left(\tilde{J_{1}}\right)+d_{2} k \tilde{J}_{1}\left(1+\tilde{J_{1}}\right)\right)-\mu \tilde{J_{1}} J_{2}\left(\mathbf{P}_{1}\left(\tilde{J}_{1}, J_{2}\right)-d_{2} k \tilde{J}_{1}\left(1+J_{2}\right)\right)+p \tilde{J}_{1}^{2} \mathbf{P}_{0}\left(J_{2}\right),
$$


where

$$
\mathbf{P}_{1}\left(\tilde{J}_{1}, J_{2}\right)=1-J_{2}^{2}+p+p \tilde{J}_{1}^{2}\left(J_{2}-1\right)+J_{2}(2+p)+\tilde{J}_{1}\left(1+J_{2}^{2}+2 p\right) .
$$

and for $\mathscr{D}_{b}$ :

$$
\mathscr{Q}\left(\mu, \tilde{J}_{1}, J_{2}\right)=\mu^{2} J_{2}^{2} \mathbf{Q}_{0}\left(\tilde{J}_{1}\right)+\mu \tilde{J}_{1} J_{2} \mathbf{Q}_{1}+p \tilde{J}_{1}^{2} \mathbf{P}_{0}\left(J_{2}\right) .
$$

For $\mathscr{D}_{b}$, we can neglect the surface tension. Eq.(23) is not valid if $J_{1}=p=1$ (which corresponds to a non growing cortex) but remains valid if $J_{2}=1$ (which corresponds to the case where the white matter does not grow). $\mathbf{Q}_{1}$ is explicited in the Appendix (9.2).

The cancellation of the dispersion relation $\mathscr{D}$, in the short wavelength approximation, is satisfied by imposing either $P_{0}\left(\tilde{J}_{1}\right)=0$ or $\mathscr{P}\left(\mu, \tilde{J}_{1}, J_{2}, d_{2}\right)=0$. It then exits two possibilities giving respectively the first and second bifurcation for $J_{1}$ at fixed $J_{2}$. The root of $\mathbf{P}_{0}$, for $\tilde{J}_{1}=J_{c}$, gives the first buckling threshold and reads:

$$
J_{c}=\frac{1}{3}\left(3+(54+6 \sqrt{33})^{1 / 3}+(54-6 \sqrt{33})^{1 / 3}\right) \sim 3.383 .
$$

However, in the case of a bilayer, a second threshold appears giving by the cancelation of $\mathscr{P}\left(\mu, \tilde{J}_{1}, J_{2}, d_{2}\right)=0$. This secondary instability is plotted in Fig.(2) and Fig.(3). In Fig.(3), a wrinkling can occur for $\tilde{J}_{1}$ smaller than $J_{c}$ if $J_{2}>J_{c}$ and $\mu$ smaller or of order one. If $\mu>1$, $\tilde{J}_{1}$ must be bigger than $J_{c}$ as shown by Fig.(2), independently of the value of $J_{2}$. However, since we restrict our discussion to $\mathscr{D}_{a}$, at this stage, the found wavelength is exactly zero in both cases which is the Biot's singularity. It is necessary to go beyond this threshold to reach a finite wavelength by considering both exponentials in Eq.(20). It turns out that no solution has been found for the one layer model in absence of capillarity [6]. To eliminate the singularity, one may consider a competitive energy in the variational elasticity problem such as the capillarity [6] or perhaps the coupling of two layers (and the existence of a new parameter $\mu$ ) which may induce the cancellation of Eq.(20). Indeed, known solutions have been found in material sciences with a soft substrate leading to a selection mechanism by the ratio $\mu$ without capillarity [36,65]. So there is perhaps hope to solve the Biot 's singularity without surface tension. Despite the simplicity of the model, the dispersion relation remains difficult for the analysis and we begin by considering extreme cases: very soft or stiff substrate. The reader, only interested in the biomechanics of the human brain, can go directly to Section (4.2.1) and then to Section (5) which concern the range of $\mu$ parameter smaller than one, so the human brain bilayer. For completeness, the full analysis is given here for arbitrary parameters which may concern other biological cases or brain pathologies.

4.1 The case of a very soft/very stiff substrate

\subsubsection{Very soft substrate}

This limit allows to reduce the dispersion relation Eq.(22) to an expansion in $\mu^{2}$ and $\mu$ only, giving:

$$
\mathbf{P}_{0}\left(\tilde{J_{1}}\right)\left(\mathbf{P}_{0}\left(\tilde{J_{1}}\right)-\frac{\tilde{J_{1}}}{\mu J_{2}} \mathbf{P}_{1}\left(\tilde{J_{1}}, J_{2}\right)\right) e^{2 k H p} \sim\left(\frac{1+\tilde{J_{1}}}{1-\tilde{J}_{1}}\right)^{2} \mathbf{Q}_{0}\left(\tilde{J_{1}}\right)^{2} .
$$

The critical threshold is then given by $\tilde{J}_{1} \sim J_{c}$, and the wave number is simply:

$$
k H=\frac{1}{p} \log \frac{\left(1+J_{c}\right)}{\left|J_{c}-1\right|} \frac{\mathbf{Q}_{0}\left(J_{c}\right)}{\mathbf{P}_{0}^{\prime}\left(J_{c}\right)\left(\tilde{J}-J_{c}\right)} .
$$


When $\mu$ is large, $\left(\tilde{J}-J_{c}\right)$ can be evaluated from the cancellation of the left-hand-side of Eq.(28), provided that $\tilde{\mathbf{P}}_{1}\left(J_{c}, J_{2}\right)$ is positive. This is clearly the case when $p$ is varied between $0<p<1$, the wavenumber $k$ and the buckling wavelength $\lambda$ can be derived according to:

$k H=\frac{1}{p} \log \left(\frac{2 \mu J_{2}}{J_{c}} \frac{1+J_{c}}{\left|J_{c}-1\right|} \frac{\left(3 J_{c}^{2}+1\right)}{\mathbf{P}_{1}\left(J_{c}, J_{2}\right)}\right)$ and $\lambda=(2 \pi p H) / \log \left(\frac{2 \mu J_{2}}{J_{c}} \frac{1+J_{c}}{\left|J_{c}-1\right|} \frac{\left(3 J_{c}^{2}+1\right)}{\mathbf{P}_{1}\left(J_{c}, J_{2}\right)}\right)$.

For very soft substrates, surface tension is not required to select the wavenumber. The wavelength remains proportional to $H$ and its weakness is due to the large value of $\mu$. However, the dependence in the $\mu$ values being logarithmic is weak and $k H \sim 1 / p$.

\subsubsection{Very stiff substrate}

The same kind of analysis can be achieved for very stiff substrate which means $\mu \rightarrow 0$. Expanding the dispersion relation in this limit, remaining at zero or first order in $\mu$ we have

$$
\mathbf{P}_{0}\left(\tilde{J_{1}}\right)\left(\mathbf{P}_{0}\left(J_{2}\right)-\frac{\mu J_{2}}{p \tilde{J}_{1}} \mathbf{P}_{1}\left(\tilde{J}_{1}, J_{2}\right)\right) e^{2 H k p} \sim\left(\frac{1+\tilde{J_{1}}}{1-\tilde{J}_{1}}\right)^{2} \mathbf{Q}_{0}\left(\tilde{J_{1}}\right) \mathbf{P}_{0}\left(J_{2}\right) .
$$

For this case, it seems that two bifurcations occur, one controlled by the upper layer and $\tilde{J}_{1} \sim J_{c}$, the other controlled by the bottom layer and $J_{2} \sim J_{c}$. In fact, in absence of capillarity in the upper layer $\left(d_{1}=0\right)$, only the bifurcation with $J_{2} \sim J_{c}$ seems possible if $P_{1}\left(\tilde{J}_{1}, J_{c}\right)$ remains positive in this neighborhood. However also the left-hand side vanishes for the same value of $J_{2}=J_{c}$. So the solvability condition requires in this case to expand to second order in $\mu$ Eq.(24) once $J_{2}=J_{c}+\mu \delta J_{2}$ where

$$
\delta J_{2}=\frac{J_{2} \mathbf{P}_{1}}{p \tilde{J_{1}} \mathbf{P}_{0}^{\prime}\left(J_{2}\right)}
$$

and $J_{2} \sim J_{c}$. A solution can be found if the following quantity is positive:

$$
\mathscr{R}=\mathbf{P}_{0}\left(\tilde{J_{1}}\right)\left(\frac{J_{2}^{2}}{\delta J_{2}} \mathbf{P}_{0}\left(\tilde{J}_{1}\right)-\tilde{J_{1}} \frac{\partial\left(J_{2} \mathbf{P}_{1}\right)}{\partial J_{2}}+\frac{p \tilde{J}_{1}}{2} \delta J_{2} \frac{d^{2} \mathbf{P}_{0}\left(J_{2}\right)}{d J_{2}^{2}}\right),
$$

where $J_{2}=J_{c}$. The interest of such analysis comes from the fact that we can expect lower value for $J_{1}$ if growth occurs in the sub-layer. If not, the folding is dominated by the first bifurcation, where $\tilde{J}_{1} \sim J_{c}$. Considering $1<\tilde{J}_{1}<J_{c}$, the term in parenthesis must be negative for a selection mechanism based on the growth in the substrate. A numerical exploration of this term as $\tilde{J}_{1}$ varies between 1 and $J_{c}$ gives effectively negative values so one can expect solutions without surface tension due to the substrate. We conclude that for a stiff substrate, growing with $J_{2}$ larger than $J_{c}$, the wrinkling instability still occurs but finds its origin from growth in the substrate and the wavenumber is given by:

$$
k H=\frac{1}{2 p} \log \left(\frac{2 \tilde{J}_{1}^{2}}{\mu \mathscr{R}}\left(\frac{1+J_{c}}{J_{c}-1}\right)^{2}\left(3 J_{c}^{2}+1\right) \mathbf{P}_{0}^{\prime}\left(J_{c}\right)\right) .
$$

Notice that our limit corresponds to the green curve in Fig.(3) for very small $\mu$. For intermediate values of $\mu$ only a numerical investigation is possible and it is the aim of the next paragraph. 

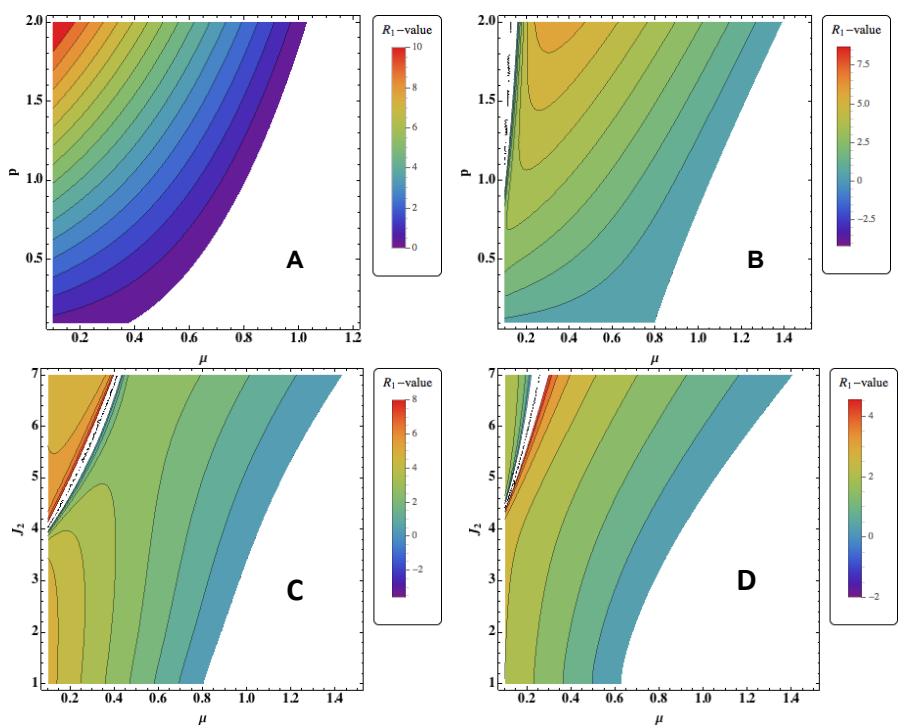

Fig. 4 Phase-diagram for the parameter $R_{1}$ (Eq.(34) for the primary bifurcation (vicinity of $J_{1} \sim J_{c}$ ) as $\mu$ and $p$ vary, for $J_{2}=1$, (no growth in the substrate, Fig.(4A)) and $J_{2}=4$ (the growth in the substrate layer exceeds the threshold of instability of a single layer, Fig.(Fig.(4B)). The same parameter as a function of the growth in the substrate for isotropic growth ( $p=1$, Fig.(4C), and, anisotropic growth $(p=0.5$, Fig.(4D)). In each figure, notice that the domains were $R_{1}>0$ is in color, with a color bar legend which indicates the magnitude of $R_{1}>0$. White area corresponds to domains where the bifurcation disappears and concerns $\mu$ values larger than one, so soft substrate.

\subsection{Intermediate $\mu$ values}

In the short-wavelength number limit, bifurcations occur when $\mathscr{D}_{a}$ vanishes. According to Eq.(22), this gives two possibilities since $\mathscr{D}_{a}$ can be decomposed into two factors. We will call first bifurcation the cancellation which occurs when the first factor vanishes, which is also the bifurcation of a single layer [6].

\subsubsection{The primary bifurcation}

The first factor in $\mathscr{D}_{a}$ does not depend on the bottom layer parameters and on $\mu$. This bifurcation is then the classical one and can only be observed if the associated wavelength has a finite non vanishing value. It is why the capillarity is considered in the model to eliminate the Biot's singularity and to determine the wavenumber $k$ or the spatial wavelength $\lambda$ of the nascent folds. If not, the $k$ value is infinite or $\lambda=0$ as explained by Biot in [11]. To solve this paradox, also called the Biot's singularity, as shown in [6], a weak surface tension coefficient is introduced, but one can observe that only the surface tension on top of the upper layer is concerned. To find the $k$ number, we expand the solvability condition Eq.(38) in the vicinity of the root of $\mathscr{D}_{a}$, given by Eq.(27), and define the key parameter $R_{1}$ :

$$
R_{1}=\frac{\mathscr{D}_{b}}{\mathscr{D}_{a}^{\prime}}=\frac{Q_{0}\left(J_{c}\right) \mathscr{Q}\left(\mu, J_{c}, J_{2}\right)}{\mathbf{P}_{0}^{\prime}\left(J_{c}\right) \mathscr{P}\left(\mu, J_{c}, J_{2}, 0\right)} \sim \frac{2\left(3 J_{c}^{2}+1\right) \mathscr{Q}\left(\mu, J_{c}, J_{2}\right)}{\left(3 J_{c}^{2}-6 J_{c}-1\right) \mathscr{P}\left(\mu, J_{c}, J_{2}, 0\right)},
$$



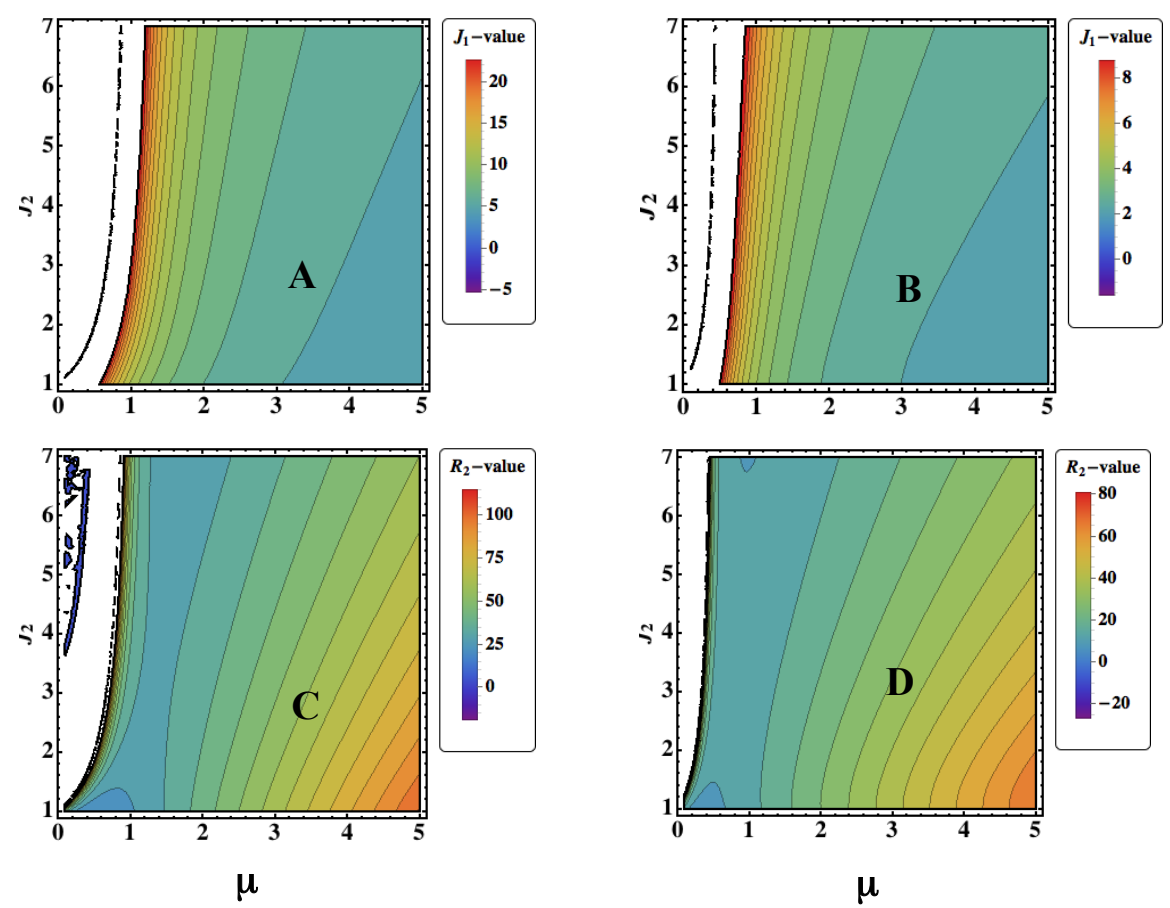

Fig. 5 Phase-diagrams for the secondary bifurcation (Section 4.2.2) as a function of the shear modulus ratio $\mu$ and the growth on the substrat $J_{2}$. Fig.(5A) and Fig.(5B) represent the threshold value for the growth in the upper layer $J_{1}$ as $\mu$ and $J_{2}$ vary. $J_{1}$ is the root of the polynomial $Q$ of Eq.(26). Notice the high values of $J_{1}$ for small $\mu$ which decreases when $\mu$ increases. This graph corresponds to Fig.(2). As shown in Fig.(5B) $(p=0.5)$, the results are very similar to Fig.(5A) but with a lower value for $J_{1}$ (Notice the change of scales in the bar legend). In Fig.(5C) and (5D) are presented the values of $R_{2}$, when it is positive, a necessary condition for folding. Despite some numerical inaccuracies, the phase diagrams A and C coincide with B and D.

where $\mathscr{D}_{a}^{\prime}$ is the derivative of $\mathscr{D}_{a}$ with respect to $\tilde{J}_{1}=J_{c}$. Only if $R_{1}$ is positive, a wavy pattern exists with a given wavelength at threshold. If not, there is no such bifurcation for $\tilde{J}_{1} \sim J_{c}$ and we must consider the secondary bifurcation corresponding to the cancellation of $\mathscr{D}_{a}$ given by $\mathscr{P}$. The numerical value of $R_{1}$ can be calculated. In Fig.(4), a phase-diagram gives $R_{1}$ when $\mu$ and $p$ vary for not growing $\left(J_{2}=1\right)$ and growing $\left(J_{2}=4\right)$ substrate (see Fig.(4A) and Fig.(4B)) while in Fig.(4C) and Fig.(4D), $\mu$ and $J_{2}$ vary with $p=1$ and $p=0.5$. Not surprisingly, the domain of folding patterns increases when $J_{2}$ increases, when the stiffness of the substrate increases and the parameter $p$ decreases. In this case, the wavenumber is:

$$
k H=\frac{1}{2 p} \log \frac{R_{1}}{\left(J-J_{c}\right)} \text { with }\left(J-J_{c}\right) \sim k d_{1} \frac{J_{c}\left(J_{c}+1\right)}{3 J_{c}^{2}-6 J_{c}-1}
$$

which leads to:

$$
k H=\frac{1}{2 p} \log \frac{2 p R_{1}\left(3 J_{c}^{2}-6 J_{c}-1\right)}{d_{1} J_{c}\left(J_{c}+1\right)} .
$$




\subsubsection{The secondary bifurcation}

We consider now the possibility to observe another bifurcation by the cancellation of $\mathscr{P}\left(\mu, \tilde{J}_{1}, J_{2}, d_{2}\right)$. The interest to study such secondary bifurcation appears clearly from the diagrams displayed in Fig.(4) which gives solutions only for rigid substrate $\mu<1$ corresponding to the analysis of Section (4.1.2). Folding seems to not exist in the white domains where $\mu$ is larger than unity. It may also happen that the cancellation of $\mathscr{P}$ occurs before the cancellation of $\mathscr{P}_{0}$, then corresponding to lower growth rates. From Fig.(2) and Fig.(3), it seems possible to reach new bifurcation parameter $J_{1}$ at a lower value than before for a growing substrate stiffer than the upper layer $(\mu<1)$. In Fig.(2), a new group of solutions for $\mu>2$ (soft substrate) and for $\tilde{J}_{1}=J_{1} / p \sim 5$ appear in the domain where there is no primary bifurcation. This limiting value is poorly dependent on the growth inside the substrate. According to our previous paragraph, one also needs to consider the sign of the new parameter $R_{2}$ given by:

$$
R_{2}=\frac{\mathscr{D}_{b}}{\mathscr{D}_{a}^{\prime}}=\frac{Q_{0}\left(J_{c}\right) \mathscr{Q}\left(\mu, J_{c}, J_{2}\right)}{\mathbf{P}_{0}\left(J_{c}\right) \mathscr{P}^{\prime}\left(\mu, J_{c}, J_{2}, 0\right)}
$$

calculated in the vicinity of the root of $\mathscr{P}\left(\mu, \tilde{J}_{1}, J_{2}, 0\right)$. However the main difficulty arises from the fact that, contrary to the primary bifurcation, the new critical growth rate $J_{t}$, (the real root of $\left.\mathscr{P}\left(\mu, \tilde{J}_{1}, J_{2}, 0\right)\right)$, is not a simple function but depends on all the other parameters such $J_{2}, \mu, p$. It is why a numerical analysis is performed. The accuracy is not perfect but gives a fast overview of the results. $\mathscr{P}$ is a polynomial of degree three in $J_{1}$ so has either one real eigenvalue or three, as shown by Fig.(2) and Fig.(3). Focussing on small $\mu$ values, according Fig.(3), for $J_{2}$ value higher than the critical threshold $J_{c}$, it exists two possible positive solutions for $J_{1} / p$ : one which is small, eventually smaller than one, the other which is higher. However these solutions do not give a positive $R_{2}$ value so are rejected in this limit. For larger values of $\mu$, Fig.(5 A) $(p=1)$ and Fig.(5 B) $(p=0.5)$ show the unique eigenvalue $J_{1}=J_{t}$ when the substrate becomes softer than the upper layer. Such solution corresponds obviously to Fig.(2). Fig.(5C) and Fig.(5D) confirm that $R_{2}>0$ quite in the same domain of existence. The wavenumber can be calculated but in this case the selection is done by the surface tension $d_{2}$ between the two layers and not by the upper layer. To conclude, the bilayer folding results mainly from the upper layer if $\mu<1$ and from the bottom layer if $\mu>1$. If $\mu<1$, (as it is believed for the brain), the analysis is simple: only the first bifurcation and the cortex layer play a role in the analysis. Instability occurs for $J_{1} \sim p 3.38$ and the wavelength scales like $\lambda \sim 4 \pi p H$.

\subsection{Comparison with pre-strain models}

In a recent work, a phase-diagram has been established with $3 D$ simulations in plain-strain elasticity [60]. Based on a pre-stretching process of the substrate, a similarity with our treatment is obvious if one takes into account only Eq.(2). However, differences occur at the level of boundary conditions. Differential growth of bilayers modifies the mechanical stresses by a factor which takes into account the volumetric growth which is different in both layers so the boundary conditions at the interface are not identical (see Appendix 9.1). Also in [60], capillarity is not introduced but, on the contrary, a delamination energy is taken into account. Boundary conditions are crucial for threshold determination and post-buckling results in the bifurcation theory. In addition, our analysis is done in two dimensions while the Wang and Zhao work involves a three-dimensional analysis. In fact the last point is not 
essential in plain-strain elasticity for uniaxial buckling, it requires simply a renormalisation of coefficients and $p g_{x}$ must be replaced by $\left(p g_{x}\right)^{2 / 3}$ on each horizontal axis. Considering $\mu=\mu_{f} / \mu_{s}$ values smaller than one, our analysis indicates that the critical parameter is $J_{1} \sim 3.383$ which gives in a $3 D$ space, a growth $g_{1} \sim 1.501$. If we transpose this growth value, the critical pre-stretch value is then $\varepsilon_{c} \sim 1-1 / g_{1} \sim 0.333$ while numerics indicates 0.35 . In absence of surface tension, singular patterns such as creases are observed. However, this limit $\varepsilon_{c} \sim 0.33$ remains a line of separation as $\mu$ increases as can be observed in the Figure (4) of [60] and in Fig.(2). So the two models recover quite analogous results in the short wavelength limits except that in our case, $J_{1}$ and $J_{2}$ can vary independently which is not the case in the pre-strain model. For infinite $\mu$ values, the results differ by a numerical factor which depends probably on the boundary conditions. The net advantage of numerical simulations is to provide informations on changes in patterns as the mismatch conditions increase so in the fully nonlinear regime.

\subsection{Two layers of arbitrary thicknesses.}

In the previous section, we have considered the dispersion relation for the wrinkling of a bilayer with an infinite substrate, an hypothesis which simplifies the analytical treatment. However, one can wonder if the ratio of thicknesses is not also a pertinent parameter for the wavelength selection. Let us come back to the more realistic case when the two layers have comparable thicknesses. The dispersion relation is then:

$$
\mathscr{D} \sim \mathscr{D}_{a}-\mathscr{D}_{b} e^{-2 H k p}-\mathscr{D}_{c} e^{-2 k},
$$

where $\mathscr{D}_{a}$ and $\mathscr{D}_{b}$ has the same meaning as before in Eq.(20) and

$\mathscr{D}_{c}=\left(\frac{1+J_{2}}{1-J_{2}}\right)^{2}\left(\mathbf{P}_{0}\left(\tilde{J_{1}}\right)-d_{1} k \tilde{J_{1}}\left(\tilde{J_{1}}+1\right)\right)\left\{\mu^{2} J_{2}^{2} \mathbf{P}_{0}\left(\tilde{J_{1}}\right)-\mu \tilde{J_{1}} J_{2} \mathbf{P}_{2}\left(\mu, J_{1}, J_{2}\right)+J_{1}^{2} p \mathbf{Q}_{0}\left(J_{2}\right)\right\}$.

In the short wavelength limit that we have considered previously, we can discard $e^{-2 k}$ if $H p$ is smaller than one so if the thickness of the upper layer multiplied by the anisotropy coefficient is smaller than the thickness of the bottom layer. In this case, the result of the previous section (for an infinite substrate) is valid. For a thin substrate, the analysis is modified although the same strategy has to be maintained. Because for the brain, the first case prevails, the cortex thickness being much smaller, we do not make the analysis for a thin substrate.

\section{Post-buckling and the gyrification index}

We now examine the buckling and post-buckling by considering the energy expansion at fourth order in the amplitude $\varepsilon$ of the profile function $F_{1}$. Since experimentally $\mu$ is smaller than one for the brain (see Table (1)), we focus on the primary bifurcation. We expect, according to our previous analysis, that the buckling threshold is given by the volumetric growth in the upper layer, with a rate value slightly larger than $p J_{c} . \varepsilon$ gives the amplitude of the buckling which must be related to the gap : $\Delta=\tilde{J}_{1}-J_{c}$. Averaging Eq.(10) and Eq.(12) per unit length, we derive to leading order in the $\Delta$ expansion:

$$
\mathscr{E}=\frac{\mu k^{3} \varepsilon^{2}}{4 \tilde{J}_{1}} \frac{\left(\tilde{J_{1}}-1\right)^{2} e^{2 H J_{1} k}\left(1+\tilde{J_{1}}\right)}{\left(1+\tilde{J}_{1}^{2}\right)^{2}}\left(-\mathbf{P}_{0}^{\prime}\left(\tilde{J_{1}}\right) \Delta+\varepsilon^{2} P_{2}\left(\tilde{J_{1}}\right) k^{4} e^{2 H k J_{1}}\right),
$$




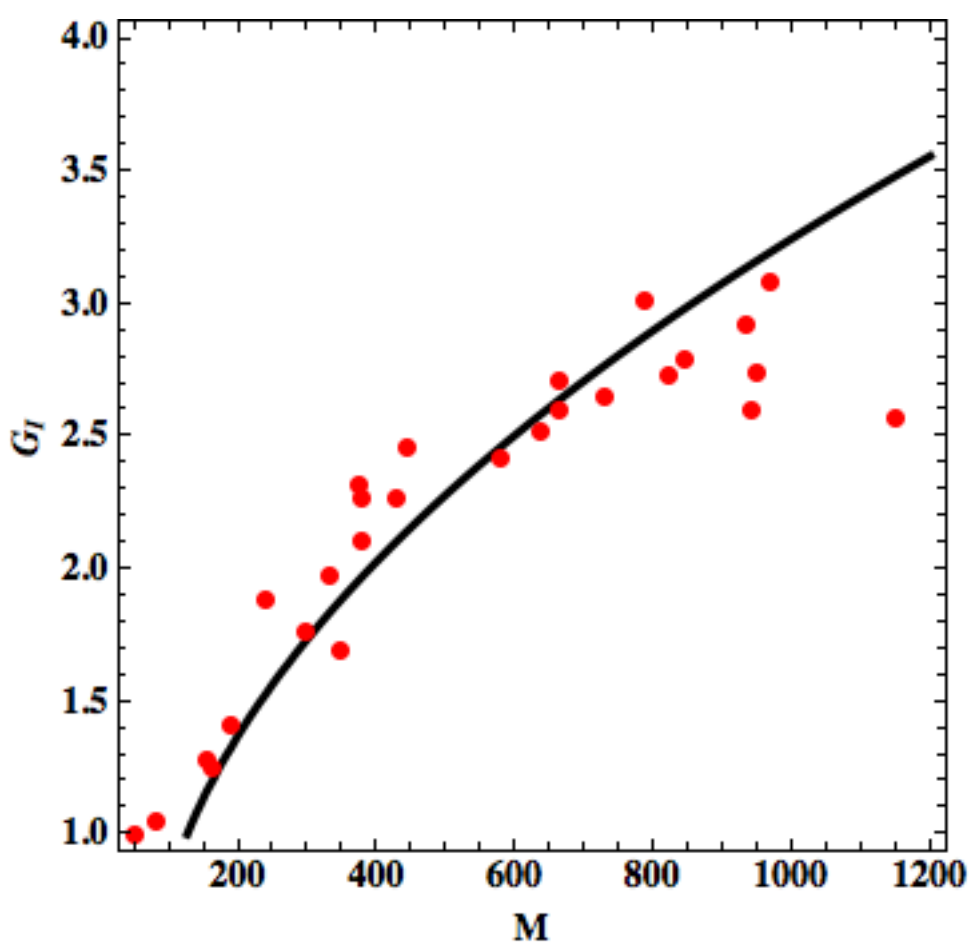

Fig. 6 The theoretical gyrification index (smooth line) and experimental data on human brains from $[68,1]$.

$$
P_{2}=\frac{\left(\tilde{J_{1}}-1\right)^{2}\left(963+1256 \tilde{J}_{1}+3265 \tilde{J}_{1}^{2}\right)}{16 p^{2}\left(\tilde{J}_{1}+1\right)^{2}\left(\tilde{J}_{1}+3\right)\left(3 \tilde{J}_{1}+1\right)\left(1+\tilde{J}_{1}\right)^{2}} .
$$

Minimization of $\mathscr{E}$ with respect to $\varepsilon$ gives the amplitude as the profile function $F_{1}(Y)$ :

$$
\varepsilon=\frac{e^{-H k J_{1}}}{k^{2}} \sqrt{\Delta} \sqrt{\frac{\mathbf{P}_{0}^{\prime}\left(\tilde{J}_{1}\right)}{2 P_{2}\left(\tilde{J}_{1}\right)}} \sim 1.3544 \frac{e^{-H k J_{1}}}{k^{2}} \sqrt{p\left(J_{1}-p J_{c}\right)} .
$$

$P_{2}$ being positive, we can conclude that the bifurcation is supercritical, the amplitude of the folds varies as the square-root of the gap from threshold $\Delta$. From Eq.(4) and Eq.(42), an evaluation of the fold amplitude can be achieved since, on top of the layer, $y_{1}$ reads:

$$
y_{1}=J_{1} H+\varepsilon e^{k J_{1} H} k \cos (k x) \sim J_{1} H+1.354 \frac{\sqrt{p\left(J_{1}-p J_{c}\right)}}{k} \cos (k x) .
$$

The quantity measured usually in brain embryogenesis is the gyrification index, ratio between the undulated surface compared to the supposed averaged surface without folds. From our model, this quantity that we call $G_{I}$ can be calculated, noticing that $y^{\prime} \sim \varepsilon e^{H J_{1} k} k^{2} \sin (k x) \sim$ $1.3544 \sqrt{p\left(J_{1}-p J_{c}\right)} \sin (k x)$,

$$
G_{I}=\frac{k}{2 \pi} \int_{-\pi / k}^{\pi / k} \sqrt{1+y^{\prime 2}} d x=\frac{1}{2 \pi} \int_{-\pi}^{\pi} \sqrt{1+m \sin (u)^{2}} d u
$$


where $m=1.3544^{2} p\left(J_{1}-p J_{c}\right)$. So the theory predicts an index which is independent of $k$ and depends only on the growth of the upper layer if the wrinkling results from the primary bifurcation. In fact it depends mainly on the the growth anisotropy coefficient $p$ and the volumetric growth $J_{1}$ at time $t$. However, our calculation results from an expansion in $\varepsilon$ of the elastic energy and so has a limited range of validity. In addition, the post-buckling analysis only gives the amplitude of the buckling but maintains the harmonic shape of the folds, which is not observed in simulations [60]. To test the range of validity of our results, we need to compare them to human brain data concerning fetus and newborns $[68,1]$ and first to calibrate $J_{1}$. The initial cortex mass of the initial configuration has to be estimated and $J_{1}$ will be the ratio between the current cortex mass to the initial one. Since we do not have a separate information for the cortex and the white matter, as a first approximation, we will take the total mass of the brain compared to its initial value. From the experimental data $[68,1]$, we estimate the constitution of the initial configuration at 23 weeks of ontogeny with a brain mass of $50 \mathrm{~g}$, and we notice that the detectable folding events occur at 28 weeks with a brain mass of $114 \mathrm{~g}$, which gives us the threshold for folding about $J_{1} \sim 114 / 50 \sim 2.28$ and an anisotropy coefficient of order $p \sim 0.7$. These informations are sufficient to derive the gyrification index according to Eq.(44) which does not take into account other physical quantities. We plot $G_{I}$ and compare it to the experimental measurements in Fig.(6). One can notice the very good agreement and, surprisingly, the model works even in a range of parameters where our formula looses its validity. Indeed our analysis includes the first nonlinearities in $\left(J_{1}-p J_{c}\right)$ and the agreement extends up to $J_{1}$ values of order 10 . For the gyrification index, the post-buckling analysis, in this case, seems to be a valuable tool allowing to reach an important quantity for the experimentalists. In addition, there is no adjustable parameter. The only required quantities to plot the theoretical $G_{I}$ curve consist of the evaluation of $J_{1}$ and the anisotropy parameter $p$. From the data $[68,1]$, the initial mass is deduced and the ratio between the brain mass at the onset of buckling and the initial mass gives us $J_{1}$, which once compared to the theory furnishes the anisotropy parameter $p \sim 0.7$. When the gyri are small and the gyrification index close to one, it is difficult to get an accurate adjustment of parameters with the formula Eq.(44). As all bifurcations in physics, the threshold is always difficult to reach experimentally. In Fig.(7), the gyrification index is displayed as a function of $J_{1}$ for different growth anisotropy coefficient, $p$ varying from 0.5 up to 1.5 . One can notice the evolution, as the growth proceeds, varying the $p$ values. In Fig.(8), a comparison with measurements made with MRI [38], close to the gyri initiation, is performed, $G_{I}$ being smaller than 1.6 while the brain mass is smaller than $200 \mathrm{~mL}$. The two data sets (blue and red points) correspond respectively to pre-term babies and fetuses after a similar period of gestation, for comparison. The data sets cover more the gyri initiation where the data are lacking in Fig.(6). We choose the best fit both for the anisotropy coefficient $p$ and the initial brain volume. For fetuses, the initial volume is estimated to be $40 \mathrm{~mL}$ (so close to the estimation of $50 \mathrm{~g}$ extracted from $[68,1]$ ). As for the anisotropy coefficient $p$, it is stronger $p \sim 0.3$ which indicates a stronger proliferation rate of the neurons along the interface between the grey and white matter in this early period of brain development. For pre-terms, the initial volume is about $27 \mathrm{~mL}$ and the anisotropy index is of order 0.55 . Then, the anisotropy coefficient is intermediate between the one of fetuses and the value extracted from the data of $[68,1]$ which cover more the last period of brain development. Here again, it seems that the early period of gyrification presents more growth anisotropy. 


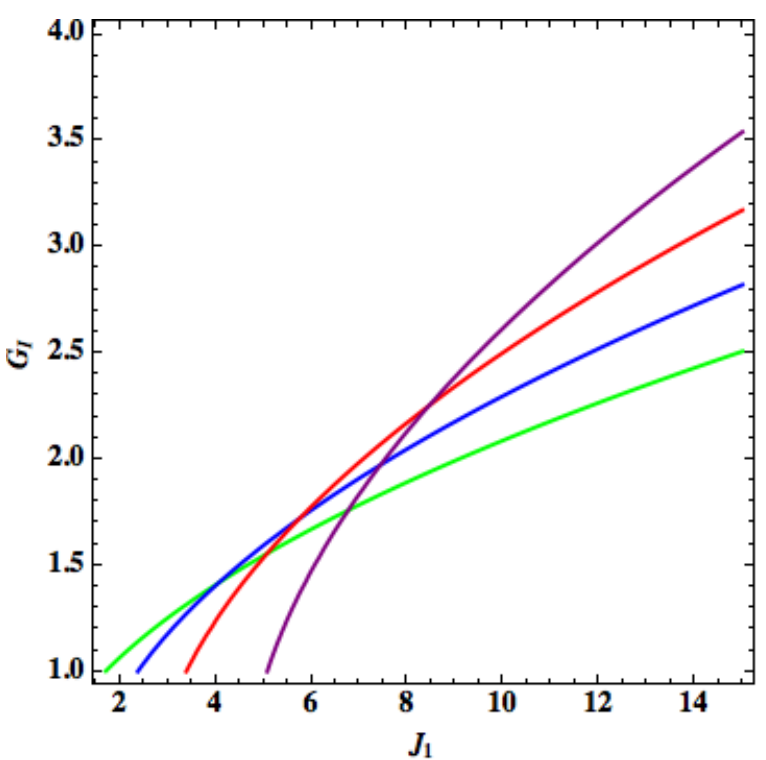

Fig. 7 Evolution of the theoretical gyrification index with the anisotropy coefficient $p=g_{y} / g_{x}$. In green, $p=0.5$, in blue $p=0.7$, in red, $p=1$, in purple $p=1.5$ (in this case, we assume the proliferation more important in the $y$ direction)

\section{Long wavelength approximation}

The aim of this section consists in recovering the classical results of material science for the wavelength selection when $\lambda \rightarrow \infty$ and is much larger than the thickness of the sample, $[10,36,65]$. This limit has been considered by Biot [10] and has been revisited in other contexts $[16,32,53]$ but not in the framework of growth. Except in [36] where the substrate is assumed neo-Hookean, usually linear elasticity is used to describe the buckling of a very thin layer put on a very soft substrate. To treat this limit, which does not concern the brain folding, we consider our dispersion relation that we expand up to $(k H)^{4}$ and keep only the leading order terms proportional to $\mu$ :

$$
\mu C_{4}(k H)^{4}-C_{2}(k H)^{2} \mu+C_{1}(k H)=0,
$$

with

$$
\left\{\begin{array}{l}
C_{4}=-(1 / 6) J_{2} p^{7}\left(\tilde{J}_{1}^{6}-3 \tilde{J}_{1}^{4}-5 \tilde{J}_{1}^{2}-1\right) \sim \frac{4}{3} J_{2} p^{7}, \\
C_{2}=J_{2} p^{5}\left(3 \tilde{J}_{1}^{4}-2 \tilde{J}_{1}^{2}-1\right) \sim 8 J_{2} p^{5} \delta J, \\
C_{1}=\left(1+J_{2}\right) p^{4}\left(1+3 \tilde{J}_{1}^{2}+\tilde{J}_{1} p-\tilde{J}_{1}^{3} p\right) \sim 4\left(1+J_{2}\right) p^{4},
\end{array}\right.
$$

where $\delta=\tilde{J}_{c}-1$. Indeed, for consistency, $\delta$ must be small and the reduced equation is then

$$
p^{3}(k H)^{3}-6 p \delta g(k H)+\frac{3\left(1+J_{2}\right)}{\mu J_{2}}=0
$$

It is worth noticing and contrary to material sciences, all coefficients depend on the growth problem, of $J_{1}$ and $J_{2}$. The imposed pressure in Biot's model is replaced by the volumetric growth $\delta g=J_{1}-1$ and the growth increase is weak in this limit. However, as in [10], 


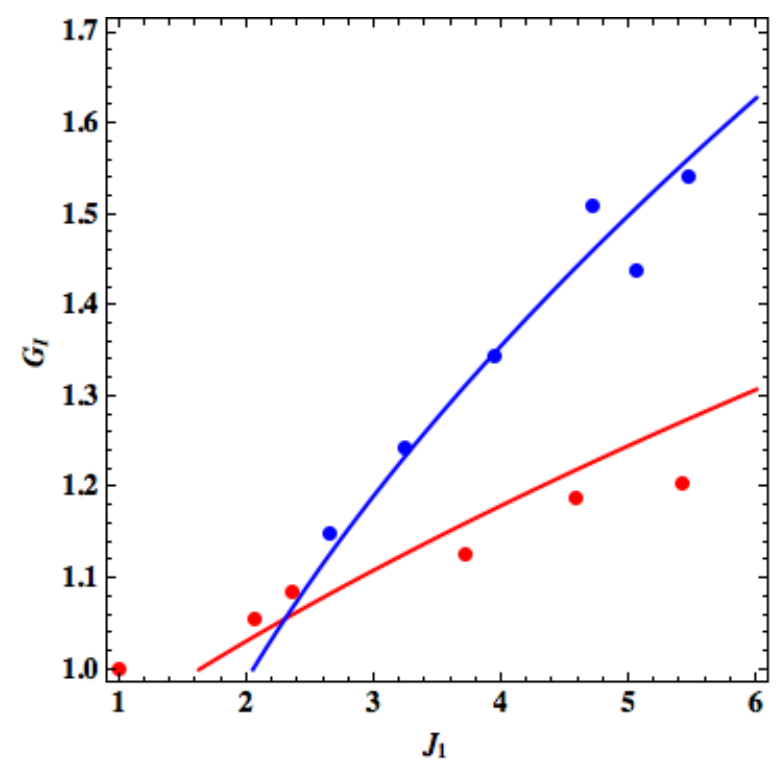

Fig. 8 Comparison of the gyrification index of fetuses obtained in utero (in red) and from pre-term babies (in blue) after the same period of gestation [38]. Comparison is made with the model and estimation of parameters is achieved via fitting.

a threshold must be reached for the buckling and we can derive easily its value and the corresponding wavelength:

$$
\delta g_{c}=\frac{1}{2}\left(\frac{3\left(1+J_{2}\right)}{2 J_{2} \mu}\right)^{2 / 3} \text { and } \lambda=2 \pi p \mu^{1 / 3} H\left(\frac{2 J_{2}}{3\left(1+J_{2}\right)}\right)^{1 / 3} .
$$

The classical results in power $1 / 3$ and $2 / 3$ is easily recovered in this limit $\mu \rightarrow \infty$.

\section{Discussion and Conclusion}

In this paper, we have examined the buckling instability of a bilayer in differential growth with the purpose to explain the brain gyrification occurring in fetal life. Our study covers the whole range of growth parameters in both layers $\left(J_{1}\right.$ and $\left.J_{2}\right)$ and stiffness ratio values $\mu$, some of them are known for the brain via previous anatomy studies and biomechanical measurements [12]. In addition, MRI imaging provides new data concerning the $G_{I}$ values $[25,38]$ in utero. Of course the complex brain geometry, even at 20 weeks of development, is very far from a double layer. It presents observable structures, not taken into account in a planar bilayer. By a careful analysis of data from $[1,68]$ concerning gyri measurements, we identify the brain growth with gyri beginning at 20/22 weeks and sulci apparition at 27 weeks. Sulci appearance is mentioned earlier in MRI studies [24,67]. The stiffness ratio has been measured by indentation measurements giving $\mu \sim 0.5$ for bovines and pigs but it presents an important regional variability [12]. If it was accepted that for the human brain, the $\mu$ value was smaller than one, a recent and careful study on cadavers post autopsy arrives to the opposite conclusion [14]. Measurements concerning fetuses, required to explain the gyri formation occurring during pregnancy, are not available. It is why our study covers the 
whole range of parameters for the $\mu$ values but our results, compared to the data $[1,68,25$, 38 ] concerning the $G_{I}$ values during pregnancy favors the hypothesis of a cortex less stiffer than the white matter.

As mentioned previously, the gyri appearance at 20/22 weeks from Fig. (6) and from the data of $[1,68]$ reveals a bifurcation occurring for a growth rate $J_{1} \sim 2.28$ which allows to identify the growth anisotropy to be $p=0.7$. Then knowing the initial thickness value $H$ which is about $0.1 \mathrm{~mm}$ at 11 weeks [46], we deduce the cortex thickness at 27 weeks : $J_{1} H \sim 0.34 \mathrm{~mm}$ and at birth $J_{1} H \sim 20 H \sim 2 \mathrm{~mm}$. Comparison with MRI studies in vitro are satisfactory since estimation from the figure (4) of [49] gives approximatively $0.5 \mathrm{~mm}$ at 27 weeks, in reasonable agreement with our zero-order approximation of purely axial growth. However, very recent measurements, also achieved with MRI $[40,41]$ indicate that the average thickness of newborn cortex is $1.99 \pm 0.1 \mathrm{~mm}$ for the left hemisphere, $2.03 \pm 0.12$ for the right hemisphere, values which will increase by $42 \%$, in the first year, much less in the second year. It must be emphasized that the cortex thickness of humans increases with aging. For example an average value of $2.59 \mathrm{~mm}$ is estimated for humans of fifty years old [62]. However, our study concerns only embryonic development and our naive estimation of $2 \mathrm{~mm}$ at birth is in perfect agrement with the last measurements [40,41]. Knowing that the size of the brain is approximatively two centimeters, at 27 weeks, we can assume the substrate infinite.

Let us now discuss the prediction of the buckling threshold according to the $\mu$ value. In the range smaller than one, we have shown that the instability originates mainly from the growth in the cortex layer. We predict the folding threshold occurring for $J_{1 c}=p 3.38 \sim 2.3$ and eventually a secondary instability occurring for $J_{2} \sim 10.9$, which is completely unrealistic at 23 days of ontogeny. So the buckling process at short wavelength is for sure due to the cortex layer. The experimental data, given in $[1,68]$, concern the whole brain growth and not separately the cortex and the white matter. Data concerning size increases, layer per layer, are only known for the pre-gyrification period, unfortunately [49]. The good agreement we have obtained for the growing cortex thickness indicates that the growth mismatch between both layers of the brain is not really significant, since we take, for the cortex, these data normalized by the initial size. Remember that $J_{1}$ is a relative growth parameter so a ratio between the current mass versus the initial mass. Clearly, the growth anisotropy $p$ and the difference between stiffnesses are much more crucial for the comparisons between the experimental and predicted gyrification index, shown in Figs. $(6,8)$.

The post-buckling treatment, at leading order of approximation, allows to theoretically evaluate the gyrification index by a simple relationship given by Eq.(44) and a rather good agreement is reached with experimental results for human brain [1,68, 25,38]. Here again, we must emphasize that if $\mu<1, G_{I}$ which evaluates the distorted surface versus the supposed smooth one only depends on the growth characteristics of the cortex. If $\mu>1$, Eq.(44) is no more valid. In our comparison, there is no adjustable parameter since the parameter $p$ as the initial size of the cortex are extracted from the data.

One can wonder how to explain pathologies such as lissencephaly or microgyria. Lissencephaly (corresponding to a lower $G_{I}$ ) value can be explained by the fact that some hypothesizes assumed in the model are not checked. For example the cortical growth looses its anisotropy, rejecting the threshold $J_{1}$ to larger values. The contrast of stiffnesses may be modified becoming larger than one. Microgyria is explained by a pathology of the meninges which consists in an increase of their thickness. Then, it can correspond to a buckling of the meninges instead of the cortex. For healthy brain, the meninges are stiffer than the cortex : they contain fibroblasts and fibers. But in pathological situation, perhaps, the observed microgyria correspond to the buckling of one of the layers of the meninges. If the meninges are thin- 
ner than the cortex at the same age, then the wavelength is expected to be smaller, giving tiny wrinkles. More informations on these pathologies are required, from the biological and physical sides, to refine the model.

To conclude, we show the complexity to find the upset of wrinkling in the short wavelength limit for bilayers with similar physical parameters. The limit where $\mu<1$ (case which gives a better agreement with experimental data for the gyrification index) is easier since the buckling answer only originates from the upper layer and the wavelength must scale with the thickness of this layer. Also the threshold for the onset of buckling can be predicted without difficulty, being approximatively $p J_{c}$. It is generally believed that the thickness of the cortex does not grow during fetal life indicating perhaps that the gyrification originates from the secondary instability. In this case, only the substrate grows and the cortex keeps its thickness constant. Numerical threshold values derived for the brain with our model shows that both thresholds are well separated and the secondary instability has no physical meaning. Our main difficulty remains the Biot's singularity and the evaluation of the microscopic length of regularization for precise comparison with experiments. In our model, the wavelength is found by a sophisticated balance between a surface energy or capillarity and the elastic energy of buckling. In the case of the brain, it is possible that the meninges, which envelop the cortex play this role as shown in Appendix 9.3 and can be considered as a surface tension. However their constitutions are complex, made of three different layers. Hopefully, the prediction of the gyrification index does not require the precise value of the wavelength.Up to an unknown constant, our estimation for the wavelength is $2 \pi p H$ so approximatively $8 \mathrm{~mm}$ for a cortex of thickness $2 \mathrm{~mm}$. To be more quantitative is difficult due to the brain geometry and to obvious structural inhomogeneities.

Finally, the growth bilayer model requires a threshold for buckling which can explain why the brain of rodent escapes to this instability. The detailed analysis of pathologies inducing modification of the cortex buckling can also be explained by modifications of the growth process. Anomalous thickening of the meninges for example may favor the buckling of this layer compared to the cortex giving a weakening of the amplitude of the gyri and a shorter wavelength, then corresponding to polymicrogyria.

\section{Acknowledgements}

This research was supported in part by Institut Universitaire de France and by the National Science Foundation under Grant No. PHY11-25915. It was motivated by enlightening discussions on folding with Ellen Kuhl and Alain Goriely in Oberwolfach session :"Mathematics of Differential Growth, Morphogenesis, and Pattern Selection" and with Oksana Manyuhina during the KITP session: "Geometry, elasticity, fluctuations and order in soft matter". MBA wants to thank the organizers: Professor Ogden and Holzapfel for their kind invitation to participate to this special issue.

\section{Appendix}

\subsection{Appendix A: Boundary conditions}

The boundary conditions concern the equality of the displacement at the interface between the two layers ( 2 conditions) and the mechanical equilibrium of both interfaces for $Y=0$ and $Y=H$ which finally gives 6 conditions. Let us focus on the interfacial conditions in 
both models: the growth and the "pre-strain" model [60], where both layers remain glued together. First we have the equality of displacements at the interface in both layers for both models, then equality of nominal stresses in the normal direction: $S_{12}$ and $S_{22}$. In the case of growing layers, it has been shown [7] that the nominal stress is given by:

$$
\mathbf{S}=G^{-1}\left(\frac{\partial E}{\partial F_{e}}-p J \mu F_{e}^{-1}\right),
$$

which gives, for neo-Hookean materials and for the upper layer, in a bidimensional approach:

$$
\mathbf{S}=\mu g_{x}\left(F_{e}-p F_{e}^{-1}\right),
$$

$p$ being the Lagrange multiplier which ensures the constraint of incompressibility. Even if we adjust correctly the pre-strain in order to represent the growth so $F_{e}$ in each layer, the boundary conditions concerning the mechanical equilibrium of the interface is different. There is no pre-factor corresponding to the growth of the sample in the pre-strain model. These pre-factors are important for bilayers with differential growth and play a role for the threshold and the wavelength selection. Let us now come back to the boundary conditions written with the stream-functions. For $Y=0, x_{1}=x_{2}$ and $y_{1}=y_{2}$ are transformed into:

$$
F_{1}(Y)=F_{2}(Y) \quad \text { and } \quad J_{2} F_{1}^{\prime}(Y)=J_{1} F_{2}^{\prime}(Y) \text { for } \quad Y=0 .
$$

Once $\mathscr{M}$ is defined as $\mathscr{M}=\mu / p \cdot\left(J_{2} / J_{1}\right)^{2}$, cancellation of the shear stress (see Chapter 5 of [45]) gives:

$$
\mathscr{M}\left(\frac{d^{2} F_{1}}{d Y^{2}}+J_{1}^{2} k^{2} F_{1}(Y)\right)=\left(\frac{d^{2} F_{2}(Y)}{d Y^{2}}+J_{2}^{2} k^{2} F_{2}(Y)\right),
$$

while the normal stress reads:

$$
\mathscr{M}\left(\frac{d^{3} F_{1}(Y)}{d Y^{3}}-k^{2}\left(p^{2}+2 J_{1}^{2}\right) \frac{d F_{1}(Y)}{d Y}\right)-J_{2}^{2} d_{2} k^{4} F_{1}(Y)=\frac{d^{3} F_{2}(Y)}{d Y^{3}}-\left(1+2 J_{2}^{2}\right) k^{2} \frac{d F_{2}(Y)}{d Y} .
$$

On top on the bilayer, for $Y=H$, Eq.(52) and Eq.(53) simplify to give:

$$
\begin{gathered}
\frac{d^{2} F_{1}}{d Y^{2}}+J_{1}^{2} k^{2} F_{1}(Y)=0, \\
\frac{d^{3} F_{1}(Y)}{d Y^{3}}-k^{2}\left(p^{2}+2 J_{1}^{2}\right) \frac{d F_{1}(Y)}{d Y}-d_{1} k^{4} F_{1}(Y)=0 .
\end{gathered}
$$

9.2 Appendix B: The polynomials of the dispersion relations

In Section 4, we have introduced the polynomial $\mathbf{P}_{1}\left(\tilde{J}_{1}, J_{2}\right)$ :

$$
\mathbf{P}_{1}\left(\tilde{J}_{1}, J_{2}\right)=1-J_{2}^{2}+p+p \tilde{J}_{1}^{2}\left(J_{2}-1\right)+J_{2}(2+p)+\tilde{J}_{1}\left(1+J_{2}^{2}+2 p\right) .
$$

Similar polynomials enter the dispersion relation, Eq.(22) and Eq.(38), that we do not explicit such as: $\mathbf{Q}_{1}$ in Eq.(26) and $\mathbf{P}_{2}$ in Eq.(39). It reads:

$$
\begin{aligned}
& \mathbf{Q}_{1}\left(\tilde{J}_{1}, J_{2}\right)=1-J_{2}^{2}-p-\tilde{J}_{1}\left(1+J_{2}^{2}-2 p\right)-J_{2}(-2+p)+p \tilde{J}_{1}^{2}\left(1-J_{2}\right), \\
& \mathbf{P}_{2}\left(\tilde{J}_{1}, J_{2}\right)=1-J_{2}^{2}+\tilde{J}_{1}\left(1+J_{2}^{2}-2 p\right)+J_{2}(-2+p)-p+p J_{1}^{2}\left(1+J_{2}\right) .
\end{aligned}
$$




\subsection{Appendix C: Skin effect and meninges}

Let us consider the case of a very thin layer on top of an infinite substrate. Only the substrate grows: $J_{1}=p=1$ and $J_{2}>1$. The dispersion relation reads at first order in $H$ :

$$
\mathscr{D}=\mathbf{P}_{0}\left(J_{2}\right)-d_{0} k J_{2}\left(1+J_{2}\right),
$$

where $d_{0}=4 H \mu$. Comparing with Eq.(22), we deduce that, in a first approximation, this system behaves like a unique layer and the very thin layer only contributes to a surface tension $d_{0}$. The meninges and more specifically the pial basement membrane, one layer among the three layers of the meninges, can be considered as a surface tension.

\section{References}

1. Armstrong, E., Scleicher, A., Omran, H., Curtis, M. \& Zilles, K.: The ontogeny of human gyrification. Cereb. Cortex 5, 5663 (1995).

2. Audoly, B. \& Boudaoud, A.: A simple buckling model for studying the emergence of herringbone patterns. Journ. Mech. Phys. Solids 56 (7): 2422-2443 (2008)

3. Barron, D.H.: An experimental analysis of some factors involved in the development of the fissure pattern of the cerebral cortex. Journal of Experimental Zoology, 113(3),553-581(1950).

4. Basan, M., Joanny, J.F., Prost, J., Risler, M.: Undulation instability of epithelial tissues. Phys. Rev. Lett. 106, 158101-04 (2011).

5. Bayly, P.V., Taber, L.A. \& Kroenke, C.D. : Mechanical forces in cerebral cortical folding: a review of measurements and models. Journ. of the mechanical behavior of biomedical materials 29, 568-581 (2014).

6. Ben Amar, M. \& Ciarletta, P.: Swelling instability of surface-attached gels as a model of tissue growth under geometric constraints. JMPS 58, 935-954 (2010).

7. Ben Amar, M. \& Goriely, A.: Growth and instability in elastic tissues. J. Mech. Phys. Solids 53, 22842319(2005).

8. Ben Amar , M. \& Jia, F. Anisotropic growth shapes intestinal tissues during embryogenesis. Proc Natl Acad Sci U S A. 110,10525-30 (2013).

9. Bender, C.M. \& Orzag, C.A. : Advanced mathematical methods for scientists and engineers. McGrawHill International Book Company

10. Biot, M.A: Folding Instability of a Layered Viscoelastic Medium under Compression.Proc R Soc A 242 (1231),444-454 (1953).

11. Biot, M.A. Surface instability of rubber in compression. Appl. sci. Res. A 12 ,168-182 (1963).

12. Budday, S., Nay R., de Rooij, R., Steinmann, P., Wyrobek, T., Ovaert,T.C. \& Kuhl, E.: Mechanical properties of gray and white matter brain tissue by indentation. J Mech Behav Biomed Mater 46, 318 330 (2015).

13. Budday,S., Kuhl, E. \& Hutchinson, J.W. :Period-doubling and period-tripling in growing bilayered systems. Philos Mag 95(28-30):,32083224 (2015).

14. Budday, S. Sommer, G., Birkl, C., Langkammer, C., Haybaeck, J., Kohnert, J. Bauer, M. Paulsen, F., Steinmann, D., Kuhl, E. \& Holzapfel, G.A. , preprint Acta Materiala (2017)

15. Brun, L., Auzias, G., Viellard, M., Villeneuve, N., Girard, N., Poinso, F., Da Fonseca, D. \& Deruelle, C.: Localized misfolding within Brocas area as a distinctive feature of autistic disorder. Biological Psychiatry: Cognitive Neuroscience and Neuroimaging 1, 160-168 (2015).

16. Cao, Y.P., Jia, F., Zhao, Y., Feng,X.Q. \& Yu, S.W. : Buckling and post-buckling of a stiff $1 \mathrm{~m}$ resting on an elastic graded substrate. International Journal of Solids and Structures 49, 16561664 (2012).

17. Carroll, M.M.: A representation theorem for volume-preserving trasformations. Int. Journ. Nonlin. Mech. 39, 219-224 (2004).

18. Clark, W \&Le Gros, E.: Deformation patterns in the cerebral cortex, Oxford University Press, Ed. John Johnson(1945).

19. Ciarletta, P. \& Ben Amar, M.: Papillary networks in the dermal-epidermal junction of the skin: A biomechanical model. Mech. Res. Com 42,68-76 (2011).

20. Ciarletta, P. \& Ben Amar, M.: Growth instabilities and folding in tubular organs: a variational method in nonlinear elasticity. Int. Journ. Nonlin Mech. 47, 248-257 (2012). 
21. Courchesne, E., Karns, C.M., Davis, H.R., Ziccardi, R., Carper, R.A., Tigue, Z.D., Chisum, H.J., Moses, P., Pierce, K. \& Lord, C. et al.: Unusual brain growth patterns in early life in patients with autistic disorder an MRI study. Neurology 57 (2), 245-254 (2001).

22. Christ, A.F., Franze, K., Gautier, H., Moshayedi, P., Fawcett, J., Franklin, R.J.M., Karadottir, R.T. \& Guck, J.: Mechanical difference between white and gray matter in the rat cerebellum measured by scanning force microscopy. J. Biomech 43 (15), 29862992 (2010).

23. Dobyns, W.B. \& Truwit, C.L: Lissencephaly and other malformations of cortical development: 1995 update. Neuropediatrics 26 (3),132-147(1995).

24. Garel, C., Chantrel,E., Brisse, H., Elmaleh, M., Luton, D., Oury, J.F., Sebag, G.,\& Hassan, M .: Fetal cerebral cortex: normal gestational landmarks identified using prenatal MR imaging. AJNR Am J Neuroradiol. 22(1),184-189 (2001).

25. Germanaud, D.,Lefvre, L., Fischer, C., Bintner, M., Curie, A., des Portes, V., Eliez, S., Elmaleh-Bergs, M., Lamblin, D., Passemard, S., Operto, G., Schaer, M., Verloes, A., Toro, R., Mangin, F.G.\& HertzPannier, L.: Simplified gyral pattern in severe developmental microcephalies? New insights from allometric modeling for spatial and spectral analysis of gyrification.NeuroImage102, 317-33 (2014).

26. Goldman, P. S. \& Galkin, T.W. : Prenatal removal of frontal association cortex in the fetal rhesus monkey: anatomical and functional consequences in postnatal life. Brain research 152(3), 451-485 (1978).

27. Goriely A., Destrade, M. \& Ben Amar, M.: Instabilities in elastomers and soft tissues. Quart. Journ. Mech. and Applied Math. 59, 615-630 (2006).

28. Goriely, A., Geers, M., Holzapfel, G. A., Jayamohan, J.,Jérusalem, A., Sivaloganathan, S., Squier, W., van Dommelen, J.A.W., Waters, S. \& Kuhl, E.: Mechanics of the brain: perspectives, challenges, and opportunities. Biomechanics and modeling in mechanobiology 14 (5), 931-965 (2015).

29. Hannezo, E., Prost, J. \& Joanny, J.F: Instabilities of monolayerd epithelia: shape and structure of villi and crypts.Phys.R ev.Lett. 107 078104-1-078105-5 (2011).

30. Holzapfel, G.A. Nonlinear solid mechanics: a continuum approach for engineering. Editor J. Wiley (2000).

31. Hong, W., Liu, Z. \& Suo, Z. : Formation of creases on the surfaces of elastomers and gels. Appl. Phys. Lett. 95, 111901-3 (2009).

32. Jia, F., Cao, Y.P., Liu, T.S., Jiang, Y., Feng, X.Q. \& Yu, S.W. : Wrinkling of a bilayer resting on a soft substrate under in-plane compression. Philosophical Magazine 92(12),1554-1568 (2012).

33. Jin, X., Zhu, F., Mao,H., Shen, M. \& Yang, K.H. : Comprehensive experimental study on material properties of human brain tissue. Journal of biomechanics 46 (16), 2795-2801 (2013).

34. Jina, L., Takeic, A. \& Hutchinson, J.W.: Mechanics of wrinkle/ridge transitions in thin film/substrate systems. JMPS 81, 22-40(2015)

35. Kaster,T., Sack, I. \& Samani, A.: Measurement of the hyperelastic properties of ex vivo brain tissue slices. Biomech.44, 1158-1163 (2011).

36. Khang, D-H, Rogers, J.\& Lee, H.H.: Mechanical buckling: Mechanics, Metrology, and Stretchable Electronics. Adv. Funct. Mater 18:1-11(2008).

37. Kucken, M. \& Newell, A.C.:Fingerprint formation. Journ Theor. Biol. 235, 71-83 (2005).

38. Lefévre,J., Germanaud,D., Dubois,J., Rousseau,F., de Macedo Santos,I.,Angleys,H., Mangin, J.F., Hppi,P.S., Girard,N. \& De Guio, F. :Are Developmental Trajectories of Cortical Folding Comparable Between Cross-sectional Datasets of Fetuses and Preterm Newborns? Cereb. Cortex 26 (7),3023-3035 (2016).

39. Li B., Cao Y.P., Feng X.Q.: Growth and surface folding of esophageal mucosa: a biomechanical model. J. Biomech. 44: 182-188 (2011).

40. Li,G., Nie,J., Wang, L., Shi, F., Gilmore, J.H, Lin, W. \& Shen, D.: Measuring the Dynamic Longitudinal Cortex Development in Infants by Reconstruction of Temporally Consistent Cortical Surfaces.Neuroimage 90, 266279 (2014).

41. Li,G., Lin,W., Gilmore, J.H. \& Shen, D.: Spatial Patterns, Longitudinal Development, and Hemispheric Asymmetries of Cortical Thickness in Infants from Birth to 2 Years of Age. The Journal of Neuroscience 35(24), 9150-9162 (2015)

42. Manyuhina, O.V., Mayett, D. \& Schwarz, J.M. :Elastic instabilities in a layered cerebral cortex: a revised axonal tension model for cortex folding. New Journal of Physics 16 (12), 123058

43. Moon, H. M. \& Wynshaw-Boris, A.: Cytoskeleton in action: lissencephaly, a neuronal migration disorder. Wiley Interdisciplinary Reviews: Developmental Biology 2(2), 229-245 (2013).

44. Ohta, H., Nordahl, C.W., Iosif, A.M., Lee, A., Rogers, S. \& Amaral, D.G. : Increased Surface Area, but not Cortical Thickness, in a Subset of Young Boys With Autism Spectrum Disorder. Autism Research, Wiley Online Library (2015).

45. Ogden, R.W. ”Non-linear elastic deformations”,Dover Publications,N.Y. (1997).

46. Rapalska, K. \& Wozniak, K. : Differentiation of the cortical plate in early human foetuses. Folia Morphol. 61(4), 233-236 (2002) 
47. Reillo, I., de Juan Romero, C., García-Cabezas, M.A. \& Borrell, V.: A role for intermediate radial glia in the tangential expansion of the mammalian cerebral cortex. Cerebral Cortex 21(7),1674-1694 (2011).

48. Rodriguez, A. K., Hoger, A. \& McCulloch, A.: Stress-dependent finite growth in soft elastic tissue. J. Biomech. 27, 455-467 (1994).

49. Scott, J.A., Habas, P.A., Kim, K., Rajagopalan, V., Hamzelou, K.S., Corbett-Detig, J.M., Barkovich, A.J., Glenn, O.A. \& Studholme, C.:Growth trajectories of the human fetal brain tissues estimated from 3D reconstructed in utero MRI. Int J Dev Neurosci. 29, 529536 (2011).

50. Siegenthaler, J. A \& Pleasure, S. J: We have got you covered: how the meninges control brain development. Current opinion in genetics \& development 21(3), 249-255, (2011).

51. Squier, W., Jansen, A.: Polymicrogyria: pathology, fetal origins and mechanisms. Acta neuropathologica communications 2(80),1-16 (2014).

52. Striedter, G. F. \& Srinivasan,S.: Knowing when to fold them,Science 349 (624),31-32(2015).

53. Sultan, E. \& Boudaoud, A.: The buckling of a swollen thin gel layer bound to a compliant substrate. J. Appl. Mech. 75, 051002 (2008).

54. Tallinen, T., Chung, J.J.Y., Biggins, J. S. \& Mahadevan, L: Gyrification from constrained cortical expansion. Proc Natl Acad Sci U S A. 111 (35),12667-12672 (2014).

55. Tallinen, T., Chung, J.J.Y., Rousseau, F., Girard N., Lefèver J. \& Mahadevan, L: On the growth and form of cortical convolutions,. Nature Physics 12, 588593(2016).

56. Toro, R. \& Burnod, Y.: A Morphogenetic model for the Development of Cortical Convolutions. Cerebral Cortex 15,1900-1913 (2005).

57. Trujillo, V., Kim J. \& Hayward, R.C: Creasing instability of surface-attached hydrogels. Soft matter 4, 564-569(2008).

58. van Dommelen, J.A.W., van der Sande, T.P.J., Hrapko, M. \& Peters, G.W.M.: Mechanical properties of brain tissue by indentation: Interregional variation. J.Mech. Behav. Biomed. Mater. 3,158-166 (2010).

59. Volpe, J.J. : Neurology of the newborn, Elsevier Health Sciences (2008).

60. Wang, Q. \& Zhao, X. : A three-dimensional phase diagram of growth-induced surface instabilities.Scientific Reports5 8887 (2015).

61. Welker, W. \& Jones, E. G., Alan Peters (eds.): Cerebral Cortex: Comparative Structure and Evolution of Cerebral Cortex, Part II. Springer US (1990).

62. Winkler, A.M., Kochunovc, P., Blangerod, J., Almasyd, L., Zillese, K., Foxc, P.T., Duggiralad, R. \& Glahna,D.C. :Cortical Thickness or Grey Matter Volume? The Importance of Selecting the Phenotype for Imaging Genetics Studies. Neuroimage. 53(3), 11351146 (2010).

63. Wu, M. \& Ben Amar, M.: Modelling fibers in growing discs of soft tissues. Journ. Math. And Mech. of Solids 39 (2), 219-224 (2014)

64. Xu, G., Knutsen, A.K., Dikranian, K., Kroenke, C.D., Bayly, P. V \& Taber, L. A.: Axons pull on the brain, but tension does not drive cortical folding. Journal of biomechanical engineering, 32 (7), 0710137 (2010).

65. Yin, J.,Yagüe, J.L., Eggenspieler, D., Gleason, K.K. \& Boyce, M.C.: Surface Micropatterning: Deterministic Order in surface Micro-topologies through Sequencial Wrinkling. Adv.Mater (2012) DOI:10.1002/adma.20120937

66. Zang, J, Zhao, X., Cao, Y., Hutchinson, J.W.: Localized ridge wrinkling of stiff films on compliant substrates J. Mech. Phys. Solids 60,1265-1279 (2012).

67. Zhang, Z., Hou, Z., Lin, X., Teng, G., Meng, H., Zang, F., Fang, F. \& Liu, S.:Development of the fetal cerebral cortex in the second trimester: assessment with 7T postmortem MR imaging. AJNR Am J Neuroradiol. 34(7), 1462-1467 (2013).

68. Zilles, K., Armstrong, E., Scleicher, A. \& Kretschmann, H.: The human pattern in gyrification in the cerebral cortex. Anat. Embryol. 170, 173179 (1988). 